\title{
Analysis of conserved microRNAs in floral tissues of sexual and apomictic Boechera species
}

\author{
Samuel Amiteye ${ }^{1}$, José M Corral' ${ }^{1}$, Heiko Vogel ${ }^{2}$ and Timothy F Sharbel ${ }^{1 *}$
}

\begin{abstract}
Background: Apomixis or asexual seed formation represents a potentially important agronomic trait whose introduction into crop plants could be an effective way to fix and perpetuate a desirable genotype through successive seed generations. However, the gene regulatory pathways underlying apomixis remain unknown. In particular, the potential function of microRNAs, which are known to play crucial roles in many aspects of plant growth and development, remains to be determined with regards to the switch from sexual to apomictic reproduction.

Results: Using bioinformatics and microarray validation procedures, 51 miRNA families conserved among angiosperms were identified in Boechera. Microarray assay confirmed 15 of the miRNA families that were identified by bioinformatics techniques. 30 cDNA sequences representing 26 miRNAs could fold back into stable pre-miRNAs. 19 of these pre-miRNAs had miRNAs with Boechera-specific nucleotide substitutions (NSs). Analysis of the Gibbs free energy $(\Delta \mathrm{G})$ of these pre-miRNA stem-loops with NSs showed that the Boechera-specific miRNA NSs significantly $(p \leq 0.05)$ enhance the stability of stem-loops. Furthermore, six transcription factors, the Squamosa promoter binding protein like SPL6, SPL11 and SPL15, Myb domain protein 120 (MYB120), RELATED TO AP2.7 DNA binding (RAP2.7, TOE1 RAP2.7) and TCP family transcription factor 10 (TCP10) were found to be expressed in sexual or apomictic ovules. However, only SPL11 showed differential expression with significant $(p \leq 0.05)$ up-regulation at the megaspore mother cell (MMC) stage of ovule development in apomictic genotypes.

Conclusions: This study constitutes the first extensive insight into the conservation and expression of microRNAs in Boechera sexual and apomictic species. The miR156/157 target squamosa promoter binding protein-like 11 (SPL11) was found differentially expressed with significant $(p \leq 0.05)$ up-regulation at the MMC stage of ovule development in apomictic genotypes. The results also demonstrate that nucleotide changes in mature miRNAs significantly $(p \leq 0.05)$ enhance the thermodynamic stability of pre-miRNA stem-loops.
\end{abstract}

\section{Background}

Apomixis, or asexual reproduction through seeds, is a naturally occurring reproductive form which has been observed in more than 400 plant species. Apomictic reproduction is, however, absent in many agriculturally important crop plants [1]. It therefore represents a potentially important agricultural tool, since introduction of apomixis into crops could be an effective way to fix and propagate a given genotype for superior crop performance. Apomixis has evolved from many different

\footnotetext{
* Correspondence: sharbel@ipk-gatersleben.de

'Leibniz Institute of Plant Genetics and Crop Plant Research (IPK),

Corrensstrasse 3, D-06466 Gatersleben, Germany

Full list of author information is available at the end of the article
}

sexual taxa [2,3], although the genetic factors underlying apomictic reproduction remain unknown.

The genus Boechera (Bocher's rock cress; formerly Arabis) is monophyletic, has a basic chromosome number $x=7$ [4], and wild populations are characterized by diploid sexuals, and diploid, aneuploid, and polyploid (mostly $2 \mathrm{n}=3 \mathrm{x}=21$ ) apomicts [5]. Plants of this genus are perennial members of the Brassicaceae which are distributed throughout North America and Greenland $[4,6,7]$. The switch from sexual to apomictic reproduction has been hypothesized to arise via de-regulation of the developmental pathways originally leading to sexual seed formation [8]. As virtually all asexual plants or animals are hybrid and/or polyploid, their associated gene regulatory changes have been proposed as possible
Ciomed Central 
triggers for the switch in reproductive mode [9]. In particular, the potential function of microRNAs (miRNAs), which are known to play crucial roles in many aspects of plant development, remains to be determined with regards to the switch from sex to apomixis.

MiRNAs are 20-24 nucleotide small endogenous nonprotein-coding regulatory RNA sequences that are produced by genes distinct from the genes that they regulate. Evidence provided by Allen et al [10] and Felippes et al [11] show that some miRNAs evolved by inverted duplications of target gene sequences, whereas others originated from random sequences that either have selfcomplementarity by chance or sequences that represent highly eroded inverted duplications. Since their discovery, several miRNAs have been computationally and/or experimentally identified and characterized in different species. A number of studies have shown that miRNAs play key roles in regulatory functions of gene expression for most eukaryotes [12,13], mainly at the post-transcriptional levels $[14,15]$. Several recent findings have implicated miRNAs in a number of biological mechanisms including leaf [16], stem [15] and root growth [17], floral organ identity, control of female gamete formation and reproductive development $[18,19]$, auxin signaling [20], and biotic and abiotic stress response [13].

Biogenesis of miRNAs involves nucleolytic processing of a precursor transcript with extensive foldback structure [21-23]. miRNAs are initially expressed as part of longer transcripts that are self-complementary foldback hairpin structures termed primary miRNAs (pri-miRNAs). Pri-miRNA precursors are transcribed by miRNA genes which are mostly independent transcript units. These pri-miRNA precursors are first processed into pre-miRNAs from which miRNAs are eventually generated by the ribonuclease III nucleases and Dicer-like1 (DCL1) in plants. Subsequently, the mature single stranded miRNA is incorporated into a miRNA-induced silencing complex (miRISC) to cleave its specific target messenger RNA (mRNA), or to effect translational attenuation of its target transcript [24,25]. Plant miRNAs bind to the protein-coding region of their target mRNAs to induce target mRNA degradation via an RNAi-like mechanism where an Argonaut (AGO) protein cleaves the miRNA-mRNA duplex, thereby repressing expression of that particular mRNA [26]. It is also known that gene repression can be effected by translational inhibition through deadenylation of the 3' poly (A) tail and decapping of the 5' end in mRNAs, which leads to progressive mRNA decay and degradation $[27,28]$.

Accurate detection and expression profiling of miRNAs will enable a better understanding of their role in plant growth and development $[13,18,20]$, and could provide insights into miRNA-mediated apomictic gene regulatory mechanisms. The main approaches for miRNA identification have been widely undertaken by computational prediction, direct cloning and sequencing. Until recently, most sequence information including Expressed Sequence Tags (ESTs) or Genome Survey Sequences (GSS) used for computational prediction of miRNAs were generated by traditional Sanger sequencing methods $[29,30]$. Compared to highly conserved miRNAs, less- or non-conserved miRNAs are often expressed at lower levels, thus making their detection more daunting using small-scale sequencing. The development of next generation sequencing technology has greatly improved the capacity to identify low abundance or tissue-specific miRNAs, and has enhanced the discovery of several conserved, non-conserved or lowly expressed miRNAs through cloning and deep sequencing of small RNA and transcriptome libraries in Arabidopsis thaliana [31,32], Triticum aestivum (wheat; [33]), Solanum lycopersicum (tomato [34]), Oryza sativa (rice), Populus trichocarpa (cotton wood), and Manihot esculenta (Cassava) [35-37]. To date, many varieties of miRNAs are reported in plants, animals, and even microbes [38].

Although miRNAs have been studied in plants for years, no extensive study has yet been performed on Boechera. The objective of this work was thus to identify and completely catalogue conserved plant miRNAs, and to compare the expression pattern of their target genes in the floral tissues of sexual and apomictic Boechera, in order to shed light on the potential role of miRNAs in the switch from sexual to apomictic reproduction. To do so we have cloned, sequenced and validated conserved miRNAs using bioinformatics and microarray techniques, and have analyzed these data using sexual and apomictic EST libraries (sequenced using 454 FLX technology) and comparative expression profiles between microdissected ovules from sexual and apomictic genotypes $[39,40]$.

\section{Results and Discussion}

\section{Homology of miRNAs to Boechera ESTs}

The BLASTn search using a reference set of 8433 nonredundant known conserved plant miRNAs against flower-specific sexual and apomictic Boechera EST libraries led to the identification of 282 sexual and 301 apomictic transcripts with high homology to miRNAs of other plant species (Figure 1). Of these, 13 sexual and 16 apomictic transcripts could fold back into stable hairpins containing conserved miRNAs (Table 1 \&2; Additional file 1, Figure S1). Many EST sequences were found that could not fold back into stem loops, although it is unclear whether this was due to the fact that they were not pre-miRNAs or whether this was due to sequencing errors introduced by the 454 FLX system. 


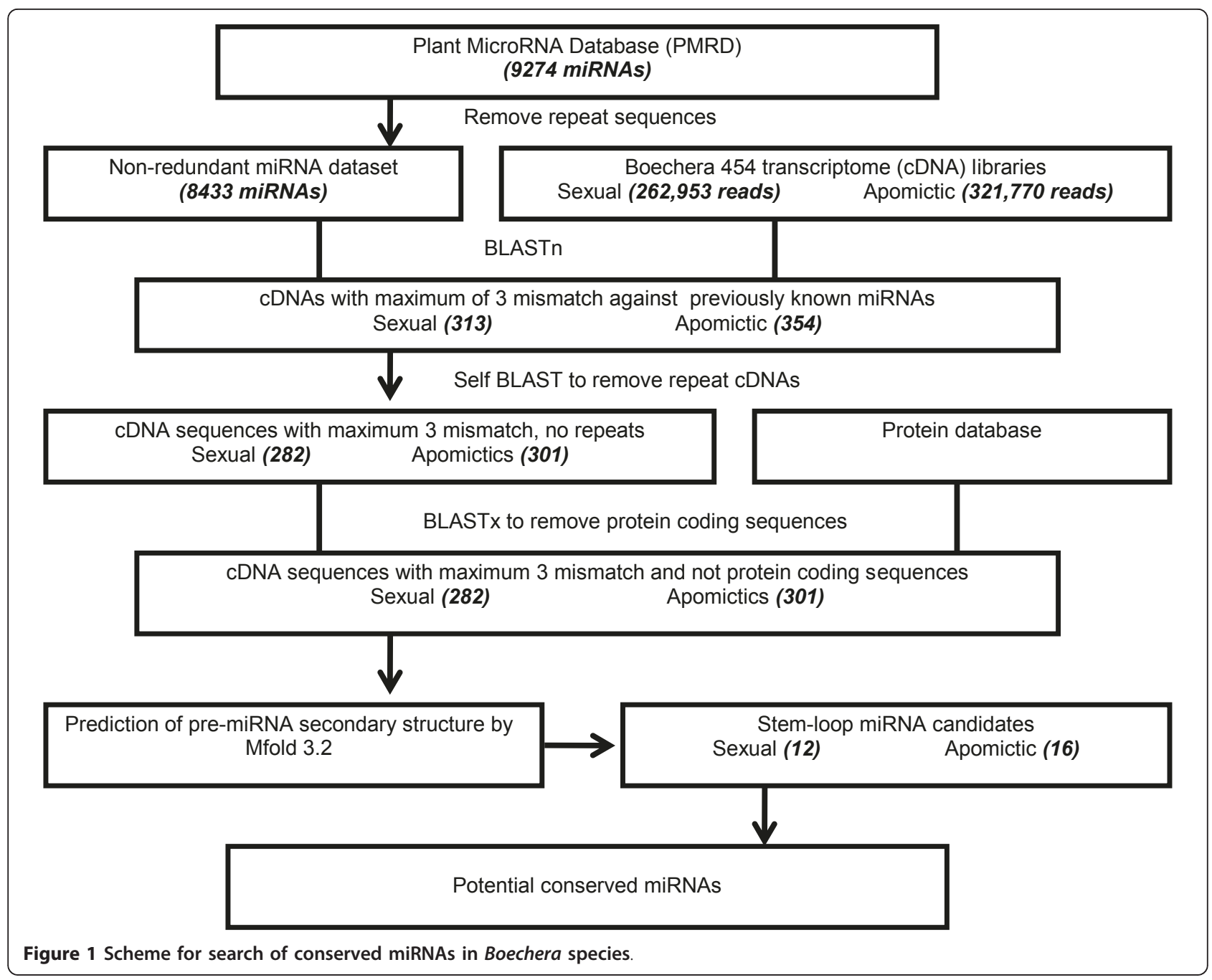

Predominantly, the less conserved miRNA families (e.g. miR444 to miR869) matched a small number of cDNA sequences which in most cases were found to be truncated precursor sequences in the EST libraries, and thus could not fold into stable stem-loops (Table 1 \&2).

\section{Bioinformatically-identified conserved miRNA families}

In all, 44 miRNA families across 67 plant species were found to match at least one Boechera 454 EST read, with $A$. thaliana being the predominant species (Figure 2). Conserved plant miRNA families in Boechera were identified to a large extent based upon high homology with reported conserved $A$. thaliana miRNAs (Figure 2). In cases where Boechera and A. thaliana did not share particular miRNA families, a search for conserved miRNA families was performed in other plant species. The predominant miRNA families which shared similarity with the highest number of Boechera 454 reads were miR156, miR157, miR160, miR167 and miR172 (Figure $3)$. It was observed also that the Boechera miRNAs exhibit a wide variation in the length of pre-miRNA sequences (Table 1 \&2; Figure 4, 5, 6 \&7). 29 families were found to be common between the sexual and apomictic genotypes. Of these, 17 mature miRNAs (miR156, 160, 167, 170, 172, 395, 396, 408, 415, 529, $824,835,841,846,859,860$ and 865) were similar in sequence, whereas 12 were different in sequence constitution due to nucleotide differences between the two reproductive modes. These included miRNAs miR157, 159, 161,166, 319, 394, 398, 400, 414, 854, 861 and 869 (Table 1 \&2). Pre-miRNA lengths varied from 66 to 233 nucleotides, with most between 66 and 184 nucleotides, a length similar to that of pre-miRNAs in other species. The location of the mature miRNAs in the precursor pre-miRNAs also varied among the miRNA families. In 12 pre-miRNAs, the miRNAs were found in the 3' arm while 18 were in the 5 ' arm of the stem-loop hairpin structures (Table 1 \&2; Figure 4, 5, 6 \&7).

Evaluation of the pre-miRNAs was also based on A $+\mathrm{U}$ content. The miRNA precursors have $\mathrm{A}+\mathrm{U}$ content 
Table 1 Characteristics of conserved miRNA families and stem-loops in sexual Boechera genotypes

\begin{tabular}{|c|c|c|c|c|c|c|c|c|c|}
\hline miRNA family & Mature miRNAs & Plant sp., NSs & NN & ARM & $\mathrm{A}+\mathrm{U} \%$ & AMFE & MFEI & EST ID & EMBL No. \\
\hline miR156 & UGACAGAAGAGAG $\underline{A} G A G C A C$ & Ath, $\mathbf{U} / \mathrm{A}$ & 75 & $5^{\prime}$ & 54.67 & 28.80 & 0.635 & ET5PU7E02HBOCM & FR869734 \\
\hline miR157 & UUGACAGAAGAGAG $\underline{-}$ GAGCAC & Sbi, U/A & 75 & $5^{\prime}$ & 54.67 & 28.80 & 0.635 & ET5PU7E02HBOCM & FR869734 \\
\hline miR159 & UUUGG $\underline{U} \cup \cup G A A G G \underline{A} A G C \cup C \cup A$ & Ath, $\mathbf{A} / \mathrm{U}, \mathbf{G} / \mathrm{A}$ & - & - & - & - & - & ETM6Q5C04I3XE2 $^{\mathbf{a}}$ & FR869757 \\
\hline miR160 & UGCCUGGCUCCCUGUAUGCCA & Ath & - & - & - & - & - & ET5PU7E02JH8D| $\left.\right|^{\mathbf{a}}$ & FR869730 \\
\hline miR161 & UGAAAGUGACUACAUCGGGGU & Ath & 92 & $5^{\prime}$ & 55.43 & 24.67 & 0.554 & ET5PU7E02IM9YA & FR869722 \\
\hline miR164 & UGGAGAAGCAGGGCACGUAAA & Gar & - & - & - & - & - & ETM6Q5C04IAM5V ${ }^{\mathbf{a}}$ & FR869752 \\
\hline miR166 & CCGGACCAGGCUUCAUCCCAG & Pta & - & - & - & - & - & ET5PU7E02JKLS| ${ }^{\mathbf{a}}$ & FR869725 \\
\hline miR167 & UGAAGCUGCCAGCAUGAUCUA & Ath & 100 & $5^{\prime}$ & 60.00 & 48.20 & 1.201 & ETM6Q5C03GWWM2 & FR869745 \\
\hline miR170 & UGAUUGAGCCGCGCCAAUAUC & Ath & - & & - & - & - & ETM6Q5C03GVNOG ${ }^{\mathbf{a}}$ & FR869746 \\
\hline miR172 & AGAAUCĆ $U G A \cup G A \cup G C \cup G C A \cup$ & Ath, $\mathbf{U} / \mathrm{C}$ & - & - & - & - & - & ET5PU7E02GZHSB ${ }^{\mathbf{a}}$ & FR869721 \\
\hline miR319 & UUGGA므GAAGGGAGCUCC $\underline{A C}$ & Ath, $\mathbf{A} / \mathrm{C}, \mathbf{U} / \mathrm{A}, \mathbf{U} / \mathbf{C}$ & - & - & - & - & - & ETM6Q5C03FYJ8Ya & FR869740 \\
\hline miR394 & UUGGCAUUCUGUCCACCUCC & Ath & 116 & $5^{\prime}$ & 57.76 & 46.46 & 1.100 & ET5PU7E02IYKJ5 & FR869733 \\
\hline miR395 & 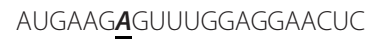 & Osa, U/A & - & - & - & - & - & ETM6Q5C03FTE98 ${ }^{\mathbf{a}}$ & FR869741 \\
\hline miR396 & UCCACAGGCUUUCUUGAACGG & Ghr & 143 & $5^{\prime}$ & 41.96 & 38.37 & 0.661 & ET5PU7E02GM4DS & FR869731 \\
\hline miR398 & UGUG $\underline{A} \cup C \cup C A G G \cup \underline{A} A C C C C \cup \cup$ & Ath, $\mathbf{U} / \mathrm{A}, \mathbf{C} / \mathrm{A}$ & - & - & - & - & - & ETM6Q5C04IDRCB $^{a}$ & FR869755 \\
\hline miR399 & 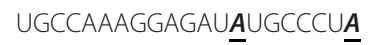 & Ath, $\mathbf{U} / \mathrm{A}, \mathbf{G} / \mathrm{A}$ & - & - & - & - & - & ET5PU7E02FZ073 ${ }^{\mathbf{a}}$ & FR869726 \\
\hline miR400 & 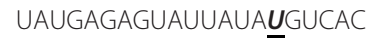 & Ath, $\mathbf{A} / \mathrm{U}$ & 76 & $3^{\prime}$ & 60.53 & 15.79 & 0.400 & ET5PU7E02JJRIA & FR869735 \\
\hline miR403 & 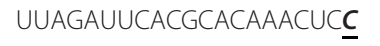 & Ath, $\mathbf{G} / \mathrm{C}$ & 75 & $5^{\prime}$ & 57.33 & 24.93 & 0.584 & ET5PU7E0213RXE & FR869723 \\
\hline miR408 & AUGCACUGCCUCUUCCCUGGC & Ath & 148 & $3^{\prime}$ & 58.78 & 33.58 & 0.815 & ETM6Q5C04JX15C & FR869749 \\
\hline miR414 & 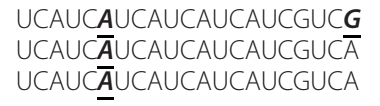 & $\begin{array}{l}\text { Ath, } \mathbf{U} / \mathrm{A}, \mathbf{A} / \mathrm{G} \\
\text { Ath, } \mathbf{U} / \mathrm{A} \\
\text { Ath, } \mathbf{U} / \mathbf{A}\end{array}$ & $\begin{array}{l}170 \\
221 \\
233\end{array}$ & $\begin{array}{l}5^{\prime} \\
3^{\prime} \\
3^{\prime}\end{array}$ & $\begin{array}{l}51.18 \\
51.01 \\
56.65\end{array}$ & $\begin{array}{l}29.29 \\
23.62 \\
24.64\end{array}$ & $\begin{array}{l}0.600 \\
0.482 \\
0.568\end{array}$ & $\begin{array}{l}\text { ET5PU7E02IZWR4 } \\
\text { ET5PU7E02GNU3F } \\
\text { ET5PU7E02114IE }\end{array}$ & $\begin{array}{l}\text { FR869738 } \\
\text { FR869727 } \\
\text { FR869724 }\end{array}$ \\
\hline miR415 & $\underline{\boldsymbol{G} A C A G A \bar{A} G \underline{A} A G A A A C A G A A C A U}$ & Ath, $\mathbf{A} / \mathrm{G}, \mathbf{C} / \mathrm{A}$ & - & - & - & - & - & ETM6Q5C03FIL8C & FR869748 \\
\hline miR444 & UUGCUGCCUCAAGCU $\underline{C} C C \underline{G} G C$ & Zma, $\mathbf{U} / \mathrm{C}, \mathbf{U} / \mathrm{G}$ & - & - & - & - & - & ETM6Q5C04IXD3L ${ }^{\mathbf{a}}$ & FR869750 \\
\hline miR482 & UCUUCCCUACACCGGCCAŪAC & Gso, U/G & - & - & - & - & - & ET5PU7E02HNZGla & FR869720 \\
\hline miR529 & $\underline{G} \subset \underline{C} \cup \underline{U} \subset \subset C C \cup C \cup C \cup C \cup \cup C \cup \cup C$ & Osa, C/G, G/C, A/U & - & - & - & - & - & ET5PU7E02HC551 ${ }^{\text {a }}$ & FR869729 \\
\hline miR824 & $\bar{U} A G \overline{A C C} A \cup \cup U G \cup G A G A A G \underline{A} G A$ & Ath, $\mathbf{G} / \mathrm{A}$ & - & - & - & - & - & ETM6Q5C04ISA8K ${ }^{\mathrm{a}}$ & FR869754 \\
\hline miR835 & UU $\underline{U} \cup \cup \underline{C} C A \cup A \cup G \cup \cup C \cup \cup \cup A \cup C$ & Ath, $\mathbf{C} / \mathrm{U}, \mathbf{G} / \mathrm{C}$ & - & - & - & - & - & ETM6Q5C04JNEVJ ${ }^{\mathbf{a}}$ & FR869751 \\
\hline miR838 & 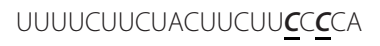 & Ath, $\mathbf{G} / \mathrm{C}, \mathbf{A} / \mathrm{C}$ & - & - & - & - & - & ETM6Q5C03FOEE6 $^{\mathbf{a}}$ & FR869747 \\
\hline miR841 & UACGACCCACU $\underline{G}$ GAAACUGAA & Ath, $\mathbf{G} / \mathrm{C}, \mathbf{U} / \mathrm{G}$ & - & - & - & - & - & ETM6Q5C03HCEIS $^{\mathbf{a}}$ & FR869742 \\
\hline miR845 & UAGCUCUGAUACCAA $\underline{A} \cup G A \cup A$ & Vvi, U/A & - & - & - & - & - & ET5PU7E02F58WOa & FR869732 \\
\hline miR846 & 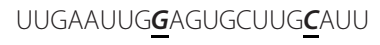 & Ath, $\mathbf{A} / \mathrm{G}, \mathbf{A} / \mathrm{C}$ & - & - & - & - & - & ETM6Q5C03FWWRY ${ }^{\mathbf{a}}$ & FR869743 \\
\hline miR852 & 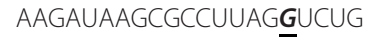 & Ath, $\mathbf{U} / \mathrm{G}$ & 89 & $5^{\prime}$ & 62.92 & 38.31 & 1.033 & ETM6Q5C03G2GJO & FR869744 \\
\hline miR854 & GAUGAGGA $\underline{G} A \underline{A} G G A G G A G G A G$ & Ath, $\mathbf{U} / \mathrm{G}, \mathbf{G} / \mathrm{A}$ & - & - & - & - & - & ETM6Q5C04JC8OP & FR869756 \\
\hline miR859 & UCUCUCUGUUGUGAA $\underline{A} \cup C A A A$ & Ath, $\mathbf{G} /$ A & - & - & - & - & - & ET5PU7E02GYEM5 ${ }^{\mathbf{a}}$ & FR869736 \\
\hline miR860 & UCA $\underline{G} \cup A G \underline{C} \cup \cup G G A C \cup \bar{A} \cup G \cup A U$ & Ath, $\mathbf{A} / \mathrm{G}, \mathbf{A} / \mathrm{C}$ & - & - & - & - & - & ETM6Q5C03G8ZLD ${ }^{\mathbf{a}}$ & FR869739 \\
\hline miR861 & CCUUGGAGAAAUAUGCQU UCAA & Ath, $\mathbf{G} / \cup$ & - & - & - & - & - & ET5PU7E02IMVAL ${ }^{\mathbf{a}}$ & FR869728 \\
\hline miR865 & 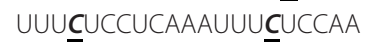 & Ath, $\mathbf{U} / \mathrm{C}, \mathbf{A} / \mathrm{C}$ & - & - & - & - & - & ETM6Q5C04JQMWDa & FR869753 \\
\hline miR869 & CAUGGUUCAAUGCA $\underline{A} G G \cup G \underline{C} \cup A$ & Gma, $\mathbf{U} / \mathrm{A}, \mathbf{U} / \mathrm{C}$ & - & - & - & - & - & ET5PU7E02JV51F ${ }^{\mathbf{a}}$ & FR869737 \\
\hline
\end{tabular}

Plant sp, NSs, Nucleotide substitutions between known plant query miRNAs and the corresponding miRNA in Boechera sexual species; NN, Number of nucleotides hairpin length; ARM, mature miRNA location in hairpin structure; AMFE, Adjusted minimum fold energy; MFEl, Minimum fold energy index; EST ID, Identifier of the 454 transcripts from which miRNA was derived. Italicized, bold and underlined red letters show nucleotide substitutions in miRNAs of Boechera sexual species. ${ }^{2}$ EST could not form secondary stem-loop structures. EMBL No., European Molecular Biology Laboratory accession number; Plant species: Ath, Arabidopsis thaliana; Gar, Gossypium arboreum; Ghr, Gossypium hirsutum; Gma, Glycine max; Gso, Glycine soja; Osa, Oryza sativa; Pta, Pinus taeda; Sbi, Sorghum bicolor; Vvi, Vitis vinifera; Zma, Zea mays.

ranging from 41.96 to $63.46 \%$ (Table 1 \&2; Figure 4, 5, 6 \&7), similar to proportions observed in other plant species [41]. Consistent with general notion, the majority of identified Boechera miRNA precursors contain more $\mathrm{A}+\mathrm{U}$ nucleotides than $\mathrm{G}+\mathrm{C}[42,43]$. It is also important to note that the formation of a stem-loop structure is not a unique feature of miRNAs, since other RNAs such as mRNA, rRNA, and tRNA can also form similar structures. For this reason, uniform systems for annotating new miRNAs comprising negative minimal fold energy (MFE), adjusted minimal fold energy (AMFE) and the minimal fold energy index (MFEI) have been developed [42-45] and have become generally accepted. Zhang et al [43] indicated that 
Table 2 Characteristics of conserved miRNA families and stem-loops in apomictic Boechera genotypes

\begin{tabular}{|c|c|c|c|c|c|c|c|c|c|}
\hline miRNA family & Mature miRNAs & Plant sp., NSs & NN & ARM & $\mathrm{A}+\mathrm{U} \%$ & AMFE & MFEI & EST ID & EMBL No. \\
\hline miR156 & $\begin{array}{l}\text { UGACAGAAGAGAGAGAGCAC } \\
\text { UGACAGAAGAGAG } \overline{\boldsymbol{A}} G A G C A C\end{array}$ & $\begin{array}{l}\text { Ath, } \mathbf{U} / \mathbf{A} \\
\text { Ath, } \mathbf{U} / \mathbf{A}\end{array}$ & $\begin{array}{c}66 \\
105\end{array}$ & $\begin{array}{l}5^{\prime} \\
5^{\prime}\end{array}$ & $\begin{array}{l}53.03 \\
52.38\end{array}$ & $\begin{array}{l}30.00 \\
25.24\end{array}$ & $\begin{array}{l}0.639 \\
0.530\end{array}$ & $\begin{array}{l}\text { ETM6Q5C01AY29E } \\
\text { ET5PU7E01BE5BP }\end{array}$ & $\begin{array}{l}\text { FR869781 } \\
\text { FR869766 }\end{array}$ \\
\hline miR157 & UUGACAGAAGAGAGAGGGCAC & Ath, $\mathbf{A} / \mathrm{G}$ & 119 & $5^{\prime}$ & 57.98 & 32.10 & 0.764 & ET5PU7E01A5S8V & FR869768 \\
\hline miR159 & UUUGGACUGAAGGGAGC̄UCCU & Ath, $\mathbf{U} / \mathrm{C}$ & - & - & - & - & - & ETM6Q5C02EBWUA ${ }^{\mathbf{a}}$ & FR869788 \\
\hline miR160 & UGCCUGGCUCCCUGUAUGCCA & Ath & 110 & $5^{\prime}$ & 58.18 & 42.10 & 1.007 & ET5PU7E01AQT2A & FR869776 \\
\hline miR161 & UCAAUGCAUUGAAAGU $\underline{A} A C \cup A$ & Ath, $\mathbf{G} /$ A & - & - & - & - & - & ETM6Q5C01 AMWRE ${ }^{\mathbf{a}}$ & FR869779 \\
\hline miR162 & UCGAUAAACCUCUGCAŪCCAG & Ptc & 84 & $3^{\prime}$ & 55.95 & 48.45 & 1.100 & ETM6Q5C01AZ870 & FR869778 \\
\hline miR166 & CCGGACCAGGCUUCAUCCCCC & Pta, $\mathbf{A} / \mathbf{C}, \mathbf{G} / \mathbf{C}$ & - & - & - & - & - & ET5PU7E01CXVM2 ${ }^{\mathbf{a}}$ & FR869765 \\
\hline miR167 & UGAAGCUGCCAGCAUGAUCUA & Ath & 100 & $5^{\prime}$ & 60.00 & 48.20 & 1.205 & ETM6Q5C01CA126 & FR869786 \\
\hline miR169 & 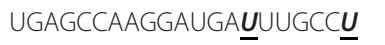 & Ath, $\mathbf{C} / \mathrm{U}, \mathbf{G} / \mathrm{U}$ & - & - & - & - & - & ETM6Q5C01B8RXC ${ }^{\mathbf{a}}$ & FR869787 \\
\hline miR170 & UGAUUGAGCCGCGCCAAUAUC & Ath & 121 & $3^{\prime}$ & 51.24 & 40.50 & 0.831 & ET5PU7E01EN973 & FR869761 \\
\hline miR172 & AGAAUCUUGAUGAUGCUGCAU & Ath & 142 & $3^{\prime}$ & 49.30 & 22.39 & 0.442 & ET5PU7E01CV6Q5 & FR869764 \\
\hline miR319 & UUGGACUGAAGGGAGCUCCUU & Ath & 184 & $3^{\prime}$ & 57.61 & 45.20 & 1.066 & ETM6Q5C02EBWUA & FR869788 \\
\hline miR394 & UUGGCAUUCUGUCAAACCUCC & Ath, $\mathbf{C} / \mathrm{A}$ & 126 & $3^{\prime}$ & 57.94 & 19.13 & 0.455 & ET5PU7E01CBSOI & FR869772 \\
\hline miR395 & AUGAAGAGUUUGGAGGAACUC & Osa, U/A & - & - & - & - & - & ETM6Q5C02DVQZ4 $^{\mathbf{a}}$ & FR869794 \\
\hline miR396 & UCCACAGGCUUUCUUGAACGG & Ghr & - & - & - & - & - & ETM6Q5C02DSTK1 $^{\mathbf{a}}$ & FR869791 \\
\hline miR398 & UGUGUUCUCAGGUCACCCCUU & Ath & - & - & - & - & - & ET5PU7E01B8LWWa & FR869770 \\
\hline miR400 & UAUGAGAGUAUUAUAGGUCAC & Ath, $\mathbf{A} / \mathrm{G}$ & - & - & - & - & - & ET5PU7E01AVMRYa & FR869771 \\
\hline miR408 & AUGCACUGCCUCUUCCCUGGC & Ath & 89 & $3^{\prime}$ & 52.81 & 39.55 & 0.838 & ET5PU7E01EE6T6 & FR869769 \\
\hline \multirow[t]{3}{*}{ miR414 } & UCAUCA $\underline{\sim} \cup C A \cup C A \cup C A \cup C G \cup C \underline{U}$ & Ath, $\mathbf{U} / \mathrm{A}, \mathbf{A} / \mathrm{U}$ & 208 & $3^{\prime}$ & 57.69 & 16.92 & 0.400 & ET5PU7E01DL36L & FR869767 \\
\hline & $\cup C A \cup C \underline{A} \cup C A \cup C A \cup C A \cup C G \cup C \underline{G}$ & Ath, $\mathbf{U} / \mathrm{A}, \mathbf{A} / \mathrm{G}$ & 170 & $5^{\prime}$ & & 29.29 & 0.603 & ET5PU7E01BXM22 & FR869762 \\
\hline & UCAUCG $\cup C A \cup C A \cup C A \cup C G \cup \overline{C A}$ & Ath, $\mathbf{U} / \mathrm{G}$ & 104 & $5^{\prime}$ & 63.46 & 29.33 & 0.803 & ET5PU7E01D5L0P & FR869759 \\
\hline miR415 & $\underline{G A C A G A G \underline{A} A G A A A C A G A A C A U}$ & Ath, $\mathbf{A} / \mathrm{G}, \mathbf{C} / \mathrm{A}$ & 135 & $5^{\prime}$ & 56.30 & 24.96 & 0.571 & ETM6Q5C01A4TW0 & FR869780 \\
\hline miR472 & UUUUGGCCUACUCCACCCCAUACC & Ath, $\mathbf{U} / \mathrm{G}, \mathbf{G} / \mathrm{A}$ & - & - & - & - & - & ETM6Q5C01B63E7 ${ }^{\mathrm{a}}$ & FR869782 \\
\hline miR529 & CUCUUECCCUCUCUCUUCUUC & Osa, $\mathbf{G} / \mathrm{C}, \mathbf{A} / \mathrm{U}$ & - & - & - & - & - & ETM6Q5C02D6DWQ $^{\mathbf{a}}$ & FR869795 \\
\hline miR776 & 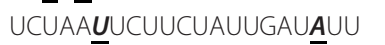 & Ath, $\mathbf{G} / \mathrm{U}, \mathbf{G} / \mathrm{A}$ & - & - & - & - & - & ET5PU7E01CU8R6 ${ }^{\mathbf{a}}$ & FR869774 \\
\hline miR820 & UCGUACUCGUGGAUGGACCAG & Osa, G/U, C/A & - & - & - & - & - & ET5PU7E01CXV4L ${ }^{\mathbf{a}}$ & FR869760 \\
\hline miR824 & UAGACCAUUUGUGAGAAG $\boldsymbol{A} G A$ & Ath, $\mathbf{G} / \mathrm{A}$ & - & - & - & - & - & ETM6Q5C01BUUMV & FR869777 \\
\hline miR835 & 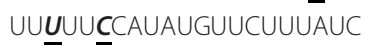 & Ath, $\mathbf{C} / \cup, \mathbf{G} / \mathrm{C}$ & - & - & - & - & - & ET5PU7E01BKF2J $^{\mathbf{a}}$ & FR869773 \\
\hline miR840 & ACACUGGAGGAGGUGAACUAA $\underline{U}$ & Ath, $\mathbf{C} / \mathrm{G}, \mathbf{A} / \mathrm{G} . \mathbf{C} / \mathrm{U}$ & - & - & - & - & - & ETM6Q5C02C26W5 $5^{\mathbf{a}}$ & FR869789 \\
\hline miR841 & UACGACCCACU $\underline{\mathbf{G}}$ GAAACUGAA & Ath, $\mathbf{G} / \mathrm{C}, \mathbf{U} / \mathrm{G}$ & - & - & - & - & - & ETM6Q5C01BolW $^{\mathbf{a}}$ & FR869785 \\
\hline miR846 & 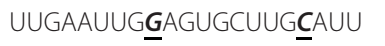 & Ath, $\mathbf{A} / \mathrm{G}, \mathbf{A} / \mathrm{C}$ & - & - & - & - & - & ETM6Q5C02D2FL9a & FR869793 \\
\hline miR854 & 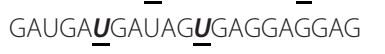 & Ath, $\mathbf{G} / \mathrm{U}, \mathbf{G} / \mathrm{U}$ & - & - & - & - & - & ETM6Q5C01A9E26 ${ }^{\mathbf{a}}$ & FR869783 \\
\hline miR857 & $\cup \cup \underline{A} \cup G \cup A \cup G \cup \cup G A A \underline{U} G \cup G \cup A \cup$ & Ath, $\mathbf{U} / \mathrm{A}, \mathbf{G} / \mathrm{U}$ & - & - & - & - & - & ETM6Q5C01AWYYJ & FR869784 \\
\hline miR859 & UCUCUCUGUUGUGAA $\underline{A} \cup C A A A$ & Ath, $\mathrm{G} / \mathrm{A}$ & - & - & - & - & - & ET5PU7E01E1HD| ${ }^{\mathbf{a}}$ & FR869775 \\
\hline miR860 & $\cup C A \underline{G} \cup A G \underline{C} \cup \cup G G A C \cup \bar{A} \cup G \cup A \cup$ & Ath, $\mathbf{A} / \mathrm{G}, \mathbf{A} / \mathrm{C}$ & - & - & - & - & - & ETM6Q5C02DJ9H7 ${ }^{\mathbf{a}}$ & FR869790 \\
\hline miR861 & 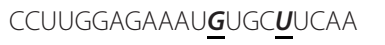 & Ath, $\mathbf{A} / \mathrm{G}, \mathbf{G} / \mathrm{U}$ & 233 & $5^{\prime}$ & 51.93 & 31.93 & 0.664 & ET5PU7E01A7VRK & FR869763 \\
\hline miR865 & UUU & Ath, $\mathbf{U} / \mathrm{C}, \mathbf{A} / \mathrm{C}$ & - & - & - & - & - & ETM6Q5C02DTC6W ${ }^{\mathbf{a}}$ & FR869792 \\
\hline miR869 & CAUGGUUCAAUGCAGGUGUUA & Gma, U/A & - & - & - & - & - & ET5PU7E01A48FH ${ }^{\mathbf{a}}$ & FR869758 \\
\hline
\end{tabular}

Plant sp, NSs, Nucleotide substitutions between known plant query miRNAs and the corresponding miRNA in Boechera apomictic species; NN, Number of nucleotides hairpin length; ARM, mature miRNA location in hairpin structure; AMFE, Adjusted minimum fold energy; MFEl, Minimum fold energy index; EST ID, Identifier of the 454 transcripts from which miRNA was derived. Italicized, bold and underlined red letters show nucleotide substitutions in miRNAs of Boechera apomictic species. ${ }^{\text {E}}$ EST could not form secondary stem-loop structures. EMBL No., European Molecular Biology Laboratory accession number; Plant species: Ath, Arabidopsis thaliana; Ghr, Gossypium hirsutum; Gma, Glycine max; Osa, Oryza sativa; Pta, Pinus taeda; Ptc, Populus trichocarpa; Sbi, Sorghum bicolor.

most identified miRNA precursors have an MFEI greater than 0.85 , which is much higher than in tRNA (0.64), rRNA (0.59), or mRNA (0.65). However, a number of pre-miRNAs with lower MFEIs have been reported, provided the number of nucleotide substitutions in the particular conserved miRNA compared with other species does not exceed three (Table 1 \&2; [41]).

\section{Microarray analysis of conserved miRNA families}

The miRNAs identified from cDNA sequencing of floral tissues, using the bioinformatics described above, were further verified using a proprietary microarray analysis with LC Sciences, in order to validate their expression in sexual and apomictic Boechera flower tissues. The LC Sciences proprietary miRNA microarray chip that was used was designed by spotting all known plant miRNAs 


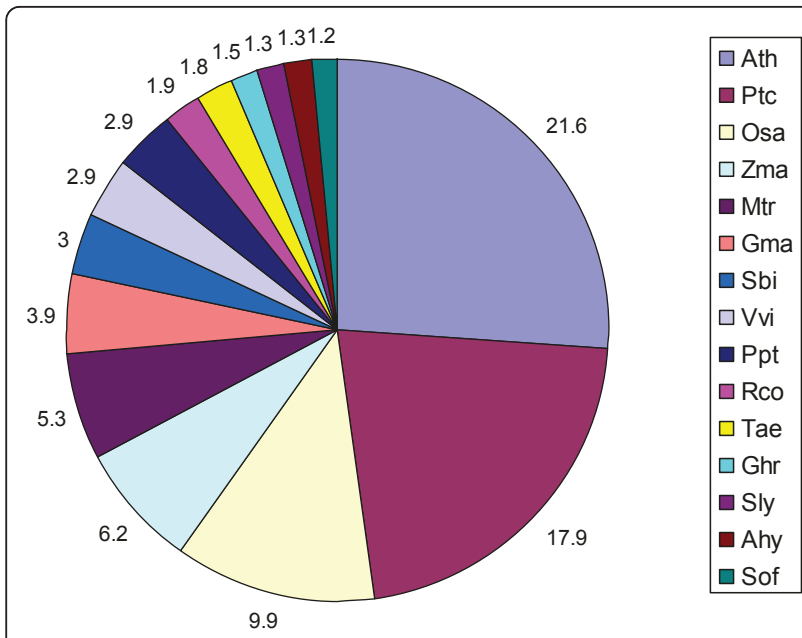

Figure 2 Percentage of conserved miRNAs between Boechera and other plant species (species with $<1 \%$ not included). Ath, Arabidopsis thaliana; Ptc, Populus trichocarpa; Osa, Oryza sativa; Zma, Zea mays; Mtr, Medicago truncatula; Gma, Glycine max; Sbi, Sorghum bicolor; Vvi, Vitis vinifera; Ppt, Physcomitrella patens; Rco, Ricinus communis; Tae, Triticum aestivum; Ghr, Gossypium hirsutum; Sly, Solanum lycopersicum; Ahy, Arachis hypogaea; Sof, Saccharum officinarum.

that were available in the miRBase Release 14 (total 1117 unique mature miRNAs) and the Plant miRNA Database, PMRD (total 5690 unique mature miRNAs). Subsequently hybridization was performed as described in Methods using isolated enriched Boechera small RNAs to confirm expressed conserved miRNAs. As expected, most $(\mathrm{n}=50)$ mature miRNAs representing 22 miRNA families were identified to be conserved mainly compared to A. thaliana. The microarray assay confirmed 15 conserved families identified with the bioinformatics techniques. It is also noteworthy that 7 and 29 other miRNA families were respectively detected

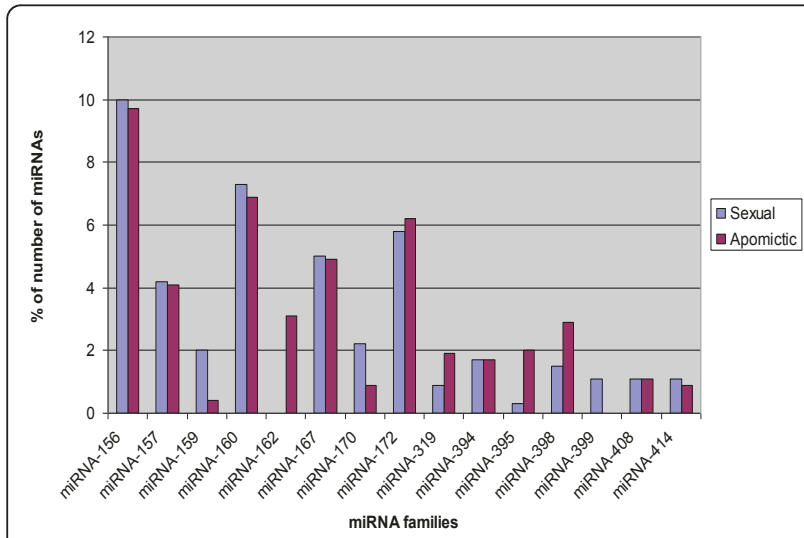

Figure 3 Abundance (based on total number of transcripts) of conserved miRNAs in Boechera species.

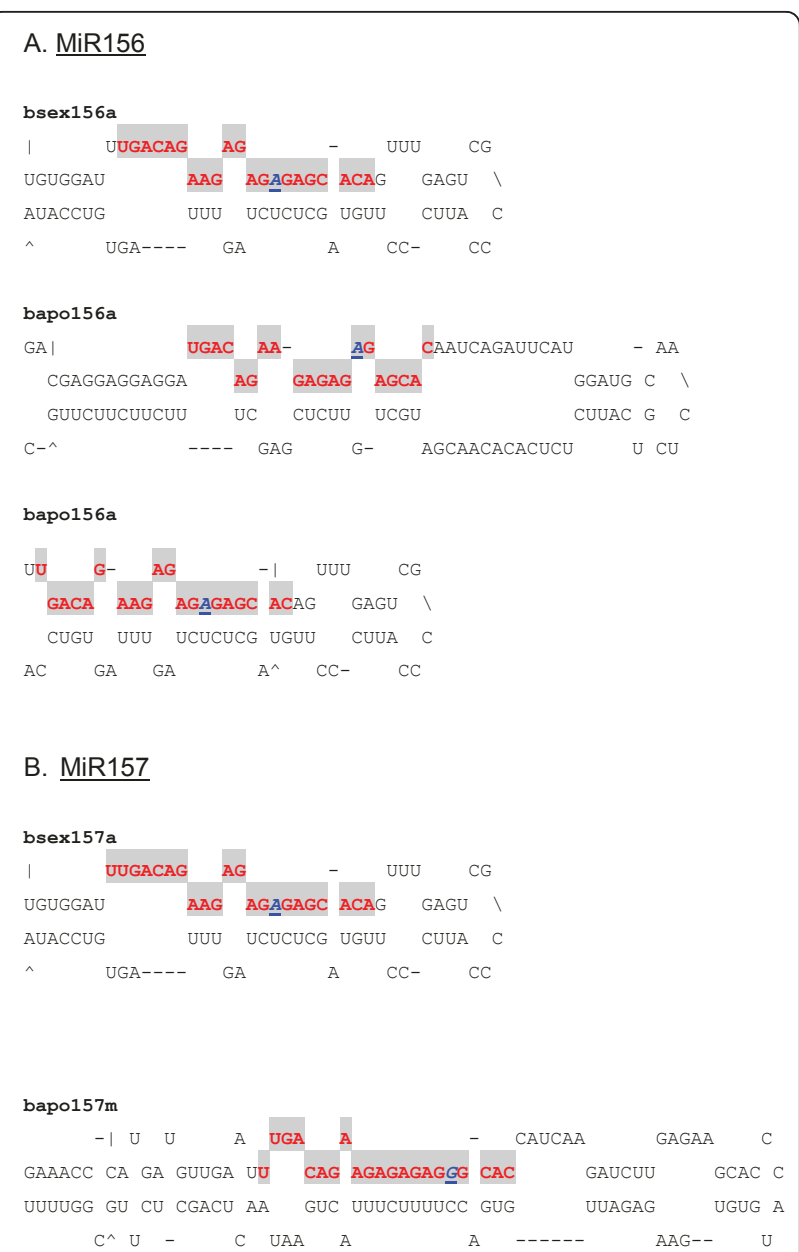

Figure 4 Predicted pre-miRNA stem-loops of miR156/157 families with nucleotide substitutions in Boechera species. Shaded red letters correspond to the sequence of the mature miRNA. Nucleotide substitutions of conserved miRNAs in other plant species compared with the corresponding miRNAs in Boechera species are shown as italicized, bold and underlined blue letters. MiRNA precursors could be slightly longer than the sequences shown in this figure.

separately by the microarray and bioinformatics approaches (Additional file 2, Figure S2).

\section{Boechera-specific miRNA nucleotide substitutions (NSs)} enhance pre-miRNA stem-loop stability

The stability of a secondary structure is quantified as the amount of free energy released or used by forming base pairs. The more negative the free energy of a structure, the more likely is formation of that structure and its stability, because more stored energy is released, and this principle is used to predict the secondary structure of a particular sequence $[46,47]$. Out of the 30 stable Boechera pre-miRNA stem-loop structures obtained, 19 contain miRNAs with nucleotide substitutions (NSs) when compared with corresponding Arabidopsis or 


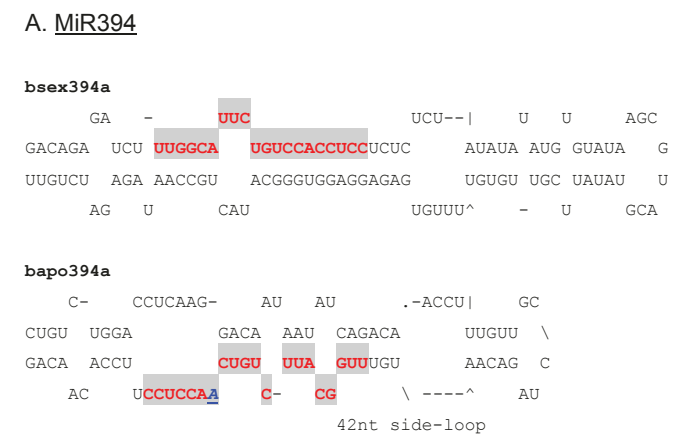

B. MiR396
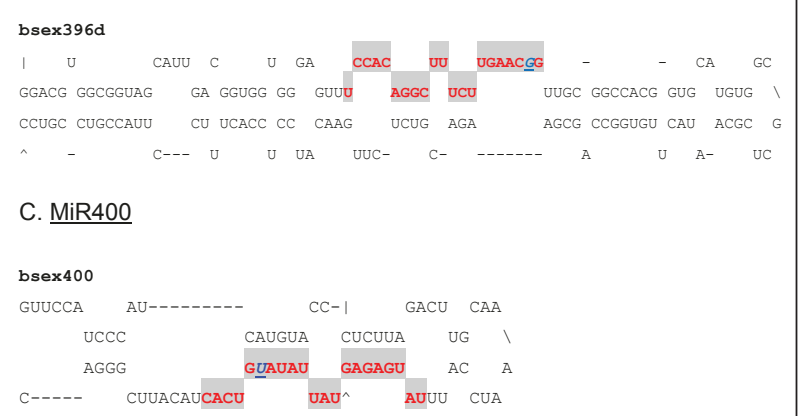

D. $\underline{\text { MiR403 }}$

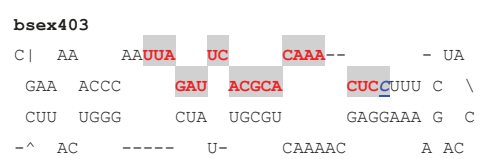

Figure 5 Predicted pre-miRNA stem-loops of miRNA families with nucleotide substitutions in Boechera species. Shaded red letters correspond to the sequence of the mature miRNA. Nucleotide substitutions of conserved miRNAs in other plant species compared with the corresponding miRNAs in Boechera species are shown as italicized, bold and underlined blue letters. MiRNA precursors could be slightly longer than the sequences shown in this figure.

other plant miRNAs. The frequency of A, C and G substitutions were similar between sexual and apomictic mature miRNAs, while $U$ appeared to show a higher rate of substitution in the apomictic mature miRNAs (Figure 8). Considering that a single nucleotide change in the sequence of a target site can affect miRNA regulation, NS could conceivably be under selection pressure to enhance the conformation and thermodynamic stability of the pre-miRNA stem-loop structure. We thus examined whether these Boechera-specific nucleotide changes had any effect on the structure and thermodynamic stability of their corresponding pre-miRNAs.

To do this, all pre-miRNAs containing miRNA NSs were selected from the various identified miRNA families (Figure 4, 5, 6 \&7). For each pre-miRNA sequence, the Gibbs free energy $(\Delta G)$ was calculated using the mfold web server [48]. Importantly, the $\Delta G$ comparison was done between a pre-miRNA with the Boechera-specific miRNA sequence, and the same premiRNA with the miRNA sequence of (mostly) Arabidopsis, in other words "correcting" the NSs in the Boechera miRNA. In most cases, the Boechera-specific premiRNA showed significantly higher $(\mathrm{p} \leq 0.05)$ thermodynamic stability (more negative free energy) than that of the pre-miRNA containing the "corrected" nucleotide substitution (Table 3). Similarly, when the corresponding pre-miRNAs in Arabidopsis/Oryza were "corrected" to Boechera miRNAs, most of the Arabidopsis/Oryza "new pre-miRNA" versions showed significantly lower ( $\mathrm{p} \leq 0.05$ ) thermodynamic stability compared to the natural pre-miRNAs (Table 4).

In all, this nucleotide substitution-stability phenomenon was most common in our analyses of both apomictic ( 8 out of 9 miRNA families) and sexual Boechera ( 5 out of 9 miRNA families; Table 3 ), in addition to Arabidopsis (9 out of 11 miRNA families; Table 4). Naturally occurring miRNA NSs thus appear to confer optimal thermodynamic stability on pre-miRNA stemloop structures in Boechera, and is consistent with similar analyses in other plants. For example, a similar comparison of the $\Delta G$ of predicted secondary structures of two variants of barley miR1137 precursor with a $C$ and a $G$ in the 13th position showed differences in stability between the variants [49]. Interestingly, Thakur et al [50] reported that species background may also be correlated with the calculation of both the minimum free energy and miRNA hairpin stability, although this difference appeared to be manifested at the level of monoand dicots. Thus, at least with respect to the comparisons between closely related Boechera and Arabidopsis used here, our data imply that natural selection has guided sequence variation in these regulatory elements.

In one case pre-miRNA stability was also manifested on the intraspecific level, comparing sexual and apomictic Boechera. In the family miR394, the pre-miRNA of the sexual Boechera species has the same miRNA sequence as in Arabidopsis, however that of the apomictic species shows one $\mathrm{C}$ to A NS change at position seven (Figure 5A). The pre-miRNA stability was examined by introducing the apomictic NS into the sexual sequence at the same position and $\Delta G$ s compared. As expected there was a decrease in the negative $\Delta \mathrm{G}$ by $6.5 \mathrm{kcal} / \mathrm{mol}$ in the "new" sexual pre-miRNA with the introduced apomictic NSs, suggesting that the sexual pre-miRNA is perhaps at its optimal thermodynamic stable state. This final evidence is consistent with trans-acting regulatory differences between sexual and apomictic ovules, the result of sequence variation in regulatory factors in the sexual 


\section{MiR414}

i. bapo414 (1)

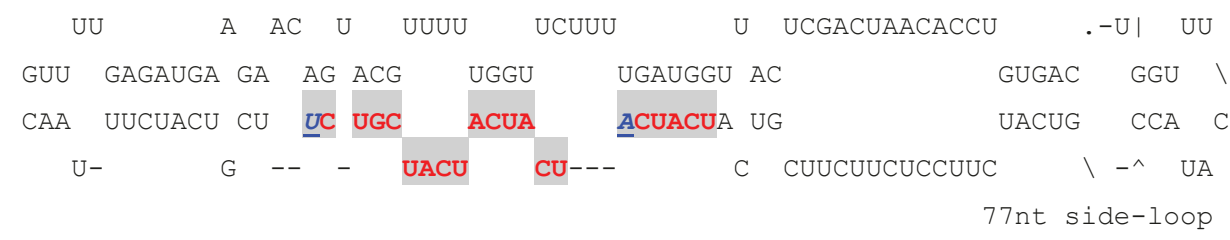

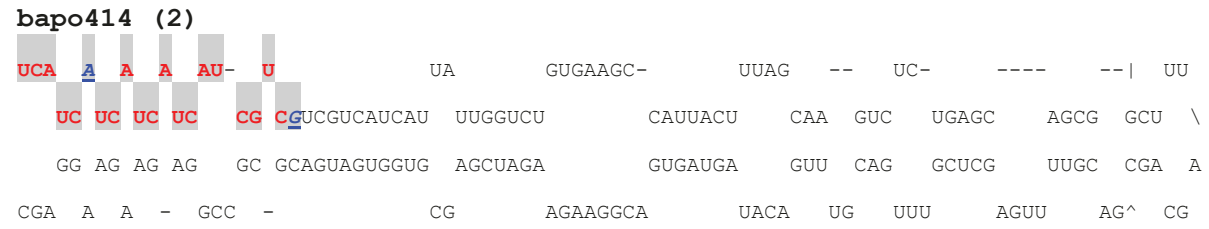

ii. $\operatorname{bsex} 414$ (1)

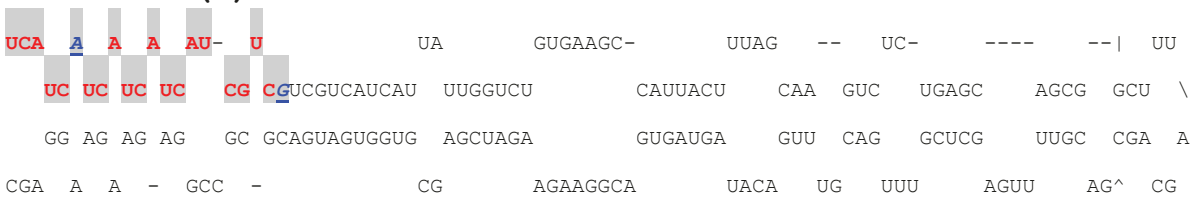

\section{bsex414 (2)}

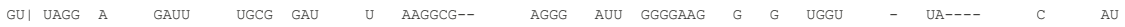

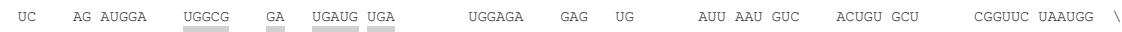

\section{$\operatorname{bsex} 414 \quad(3)$}

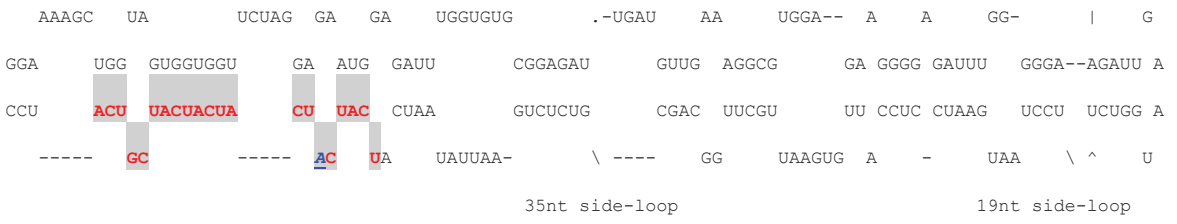

Figure 6 Predicted pre-miRNA stem-loops of miR414 family with nucleotide substitutions in Boechera species. Shaded red letters correspond to the sequence of the mature miRNA. Nucleotide substitutions of conserved miRNAs in other plant species compared with the corresponding miRNAs in Boechera species are shown as italicized, bold and underlined blue letters. MiRNA precursors could be slightly longer than the sequences shown in this figure. 

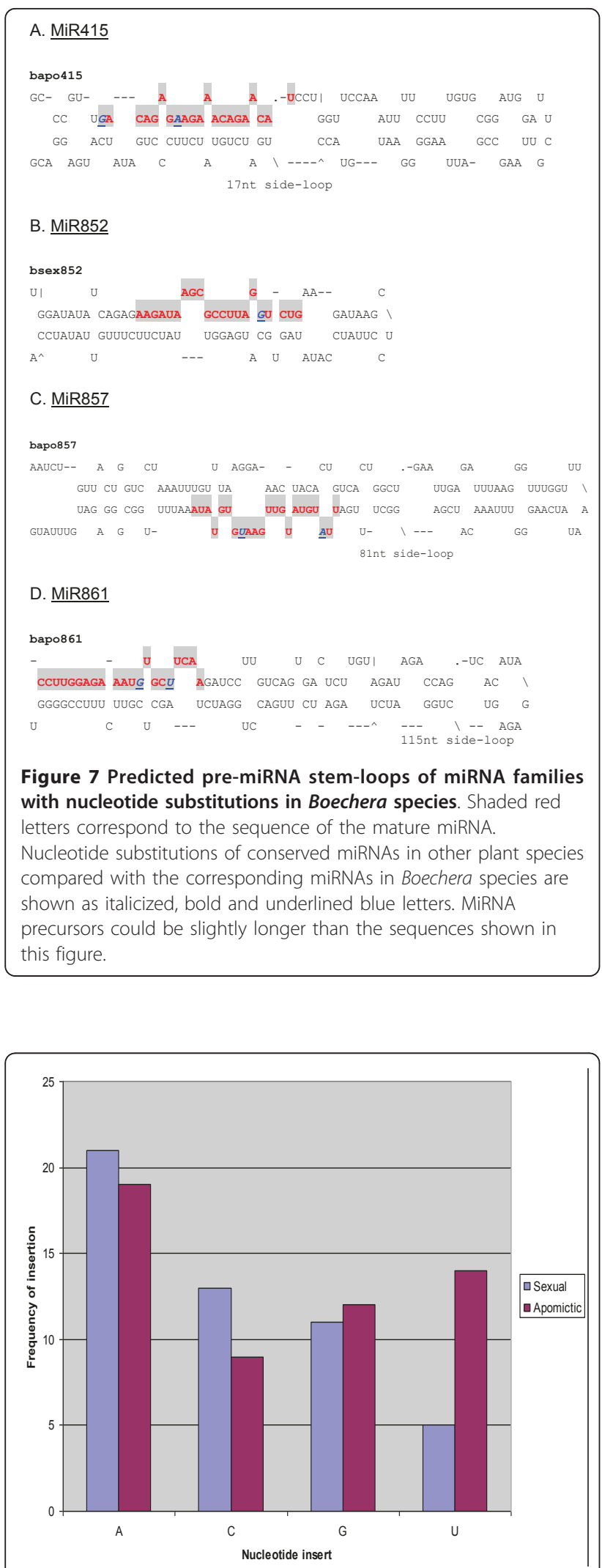

Figure 8 Frequency of nucleotide substitutions related to Arabidopsis thaliana in mature Boechera miRNAs.
Table 3 Boechera miRNA nucleotides substituted with those of Arabidopsis/Oryza

\begin{tabular}{|c|c|c|c|c|}
\hline Pre-miRNA & NSs & NN & & $\Delta \mathrm{G}$ \\
\hline & $\begin{array}{l}\text { Plant sp./ } \\
\text { Boechera }\end{array}$ & & $\begin{array}{c}\text { Natural } \\
\text { HP }\end{array}$ & $\begin{array}{c}\text { "Corrected" } \\
\text { HP }\end{array}$ \\
\hline Sexual Species & & & & \\
\hline bsex-MIR156a* & U/A & 75 & -21.6 & -19.3 \\
\hline bsex-MIR157a & U/A & 75 & -21.6 & -19.3 \\
\hline bsex-MIR396 & $U / G$ & 143 & -55.9 & -56.8 \\
\hline bsex-MIR400* & $\mathrm{A} / \mathrm{G}$ & 76 & -12.0 & -11.7 \\
\hline bsex-MIR403* & $\mathrm{G} / \mathrm{C}$ & 75 & -18.7 & -15.5 \\
\hline bsex-MIR414* (1) & $U / A, A / G$ & 170 & -49.8 & -48.2 \\
\hline bsex-MIR414 (2) & U/A & 221 & -52.2 & -56.4 \\
\hline bsex-MIR414 (3) & U/A & 233 & -57.9 & -60.0 \\
\hline bsex-MIR852 & $U / G$ & 89 & -34.1 & -34.7 \\
\hline $\begin{array}{l}\text { Apomictic } \\
\text { Species }\end{array}$ & & & & \\
\hline bapo-MIR156a*(1) & U/A & 66 & -19.8 & -15.6 \\
\hline bapo-MIR156a*(2) & U/A & 105 & -27.9 & -26.7 \\
\hline bapo-MIR157m* & $A / G$ & 119 & -38.2 & -31.3 \\
\hline bapo-MIR394a & C/A & 126 & -23.8 & -26.2 \\
\hline bapo-MIR414* (1) & $U / A, A / U$ & 208 & -35.2 & -34.2 \\
\hline bapo-MIR414* (2) & $U / A, A / G$ & 170 & -49.8 & -48.2 \\
\hline bapo-MIR414* (3) & U/G & 104 & -30.5 & -29.2 \\
\hline bapo-MIR415* & $\mathrm{A} / \mathrm{G}, \mathrm{C} / \mathrm{A}$ & 135 & -33.8 & -30.3 \\
\hline bapo-MIR861* & $\mathrm{A} / \mathrm{G}, \mathrm{G} / \mathrm{U}$ & 233 & -79.4 & -71.4 \\
\hline
\end{tabular}

HP, Hairpin; NN, Number of nucleotides hairpin length; NSs, Nucleotide substitutions. Asterisk indicates cases where "correction" of Boechera miRNA $\mathrm{NSs}$ led to less stem-loop stability due to decrease in $\Delta \mathrm{G}$. Where there are two or more pre-miRNAs with the same miRNA, they are distinguished by numbers in brackets.

(homozygous) versus apomictic (hybrid) genomes, as suggested by Sharbel et al [40].

\section{Conserved Boechera miRNAs target many transcription factors (TFs)}

The BLAST analyses here have revealed many potential regulatory gene targets. Consistent with the results of functional studies in other plant species, such as Arabidopsis, rice and corn $[26,51,52]$, the majority $(40 \%)$ of target proteins in Boechera are transcription factors (Table 5; Additional file 3 Table S1). Transcription factors (TF) have been estimated in rice to be about $70 \%$ of conserved miRNA targets, while in wheat it has been predicted to be $35 \%[33,53]$. The other targets are mostly associated with plant metabolism, development, signal transduction and response to environmental stress including cold, salinity, drought and nutritional deficiency $[35,29,54,55]$.

The EST libraries from which the Boechera miRNAs were mined were flower-specific $[39,40]$, and expectedly, a number of identified TF-targeting miRNAs have been associated with flower development in other species. For example, miR156 and miR157, the homologues of the 
Table 4 Known plant miRNA nucleotides substituted with those of Boechera

\begin{tabular}{|c|c|c|c|c|}
\hline \multirow[t]{2}{*}{ Pre-miRNA } & \multirow{2}{*}{$\begin{array}{c}\begin{array}{c}\text { Nucleotide } \\
\text { Substitutions }\end{array} \\
\text { Plant sp./Boechera }\end{array}$} & \multirow[t]{2}{*}{ NN } & \multicolumn{2}{|c|}{$\Delta \mathbf{G}$} \\
\hline & & & $\begin{array}{c}\text { Natural } \\
\text { HP }\end{array}$ & $\begin{array}{c}\text { "Corrected" } \\
\text { HP }\end{array}$ \\
\hline $\begin{array}{l}\text { ath- } \\
\text { MIR156a* }\end{array}$ & $U / A$ & 123 & -57.1 & -52.4 \\
\hline $\begin{array}{l}\text { ath- } \\
\text { MIR157m* }\end{array}$ & $\mathrm{A} / \mathrm{G}$ & 50 & -10.2 & -9.3 \\
\hline ath-MIR394* & $C / A$ & 117 & -53.1 & -46.6 \\
\hline osa-MIR396* & $U / G$ & 154 & -64.7 & -60.1 \\
\hline ath-MIR400* & $\mathrm{A} / \mathrm{U}$ & 102 & -38.4 & -34.3 \\
\hline ath-MIR403* & $\mathrm{G} / \mathrm{C}$ & 135 & -38.8 & -35.4 \\
\hline \multirow[t]{5}{*}{ ath-MIR414 } & $\mathrm{U} / \mathrm{A}, \mathrm{A} / \mathrm{G}$ & 108 & -22.0 & -22.3 \\
\hline & U/G & & -22.0 & -21.8 \\
\hline & $U / A, A / U$ & & -22.0 & -26.4 \\
\hline & $U / A$ & & -22.0 & -23.3 \\
\hline & $\mathrm{U} / \mathrm{A}, \mathrm{A} / \mathrm{G}, \mathrm{A} / \mathrm{G}$ & & -22.0 & -22.4 \\
\hline ath-MIR415* & $\mathrm{A} / \mathrm{G}, \mathrm{C} / \mathrm{A}$ & 110 & -27.0 & -24.8 \\
\hline ath-MIR852 & $U / G$ & 202 & -80.6 & -80.8 \\
\hline ath-MIR861* & $\mathrm{A} / \mathrm{G}, \mathrm{G} / \mathrm{U}$ & 132 & -56.3 & -51.6 \\
\hline
\end{tabular}

HP, Hairpin; NN, Number of nucleotides hairpin length; Asterisk indicates cases where "correction" of Arabidopsis/Oryza miRNA NSs led to less stem-loop stability.

squamosa-promoter binding proteins and whose function is well conserved across plant species [43], were identified in both apomictic and sexual Boechera (Table 5; Additional file 3, Table S1). In Arabidopsis these TF regulatory miRNAs have been reported to regulate the Antirrhinum floral meristem identity squamosa promoter binding protein-like (SPL) genes [56]. Other TF regulatory miRNA families which have regulatory roles during flower development in other species were also identified (Table 5; Additional file 3, Table S1), including miR156, miR159, miR164 and miR172, which have been implicated in the control of LFY expression, floral organ identity, and flowering time $[27,57,58]$. miR172 has furthermore been reported to regulate stem cell fate, and defines the inner boundary of the APETALA3 and PISTILLATA expression domains in Arabidopsis floral meristems [38].

A number of well-defined TF targeting miRNAs were also identified in Boechera. For example, miR160 and miR167 (Table 5; Additional file 3, Table S1) are associated with post-transcriptional regulation of the $A$. thaliana auxin response transcription factor (ARF) family genes [26,59]. miR319 is known to regulate the expression of TCP transcription factor genes whose down-regulations cause abnormalities in leaf development [16]. Vierstra [60] showed that miR394 regulates the messages of F-box proteins, which in turn target specific proteins for proteolysis by making them substrates for ubiquitination by SCF E3 ubiquitin ligases. Growth Regulating Factor genes, the targets of the miR396 family, are putative transcription factors that regulate cell expansion in leaf and cotyledon [61]. Argonaute, one of the important proteins in the regulation of miRNA biogenesis, is a target of miR403 whereas miR408 regulates a copper ion binding protein. The miR414 family regulates a number of other genes including the transcription factors, transducin family protein/WD-40 repeat family protein and peptidyl-prolyl cis-trans isomerase cyclophilin-type family protein.

\section{Expression patterns of transcription factor (TF) targets and apomixis in Boechera}

The switch from sexual to apomictic seed production is hypothesized to involve global regulatory changes during ovule development which are induced by hybridization and/or polyploidy $[9,62]$, both common characteristics of apomictic plants and parthenogenetic animals. Using data from a previously-published SuperSAGE analysis $[39,40]$, the ovule expression patterns of putative target TFs for the miRNAs identified here were compared between sexual and apomictic Boechera across four ovule developmental stages. Of the 17 TFs identified as potential miRNA targets, expression data for 6 were found in the SuperSAGE libraries, including: the squamosa promoter binding protein like SPL6, SPL11 and SPL15, Myb domain protein 120 (MYB120), RAP2.7, TOE1 RAP2.7 (RELATED TO AP2.7) DNA binding and TCP10 (TCP family transcription factor 10), which are targets of the miRNA families miR156/157, miR159, miR172 and miR319 respectively.

It is noteworthy that, whereas the other genes showed no significant differential expression levels between sexual and apomictic species, SPL11 was found to be significantly ( $p \leq 0.05)$ up-regulated at the stage two of ovule development in apomictic species in all libraries studied (Figure 9). SPL11 also showed low level expression in all the other apomictic ovule stages and at only stage two of the sexual ovules. Using six apomictic and five sexual genotypes of Boechera, the differential expression of SPL11 at ovule stage two of floral development was further validated using quantitative Real Time-PCR. With the exception of a single sexual $B$. divaricarpa from Mule Ranch, Montana, all apomictic accessions clearly showed relatively higher expression of SPL11 than the sexuals (Figure 10), result which is consistent with the expression pattern observed with the SPL11 SuperSAGE tag (Figure 9). The single sexual outlier (Figure 10) for SLP11 implies that the expression pattern of this TF may not be a key factor associated with apomixis expression, but rather is associated with DNA sequence variation in regulatory factors in the hybrid $B$. divaricarpa. Alternatively, population-level variation for 


\section{Table 5 Transcription factor targets of conserved miRNA families in Boechera species}

\begin{tabular}{|c|c|c|c|c|}
\hline miRNA family & Target protein & Function of target & Target gene (UPE) & E-value \\
\hline \multirow[t]{7}{*}{ miR156/157 } & Squamosa promoter binding protein like & & & \\
\hline & SPL11 & Transcription factor & AT1G27360 (11.430) & \\
\hline & SPL 2 & & AT5G43270 (11.987) & \\
\hline & SPL10 & & AT1G27370 (12.296) & \\
\hline & SPL15 & & AT3G57920 (14.449) & \\
\hline & SPL 9 & & AT2G42200 (16.239) & \\
\hline & SPL6 & & AT1G69170 (17.076) & \\
\hline miR159 & Myb domain protein 120 (MYB120); DNA binding & Transcription factor & AT5G55020 (7.049) & 3.5 \\
\hline miR160 & Auxin Response Factor 10 (ARF10); transcription factor & Transcription factor & AT2G28350 (18.139) & 1 \\
\hline miR167 & Auxin response factor 8 (ARF8) & Transcription factor & AT5G37020 (17.281) & 3.5 \\
\hline miR169 & CCAAT-binding transcription factor (CBF-B/NF-YA) subunit B & Transcription factor & ATIG17590 (18.910) & 2 \\
\hline MiR170/171 & Scarecrow transcription factor family protein & Transcription factor & AT3G60630 (14.202) & 1 \\
\hline miR172 & RAP2.7, TOE1 | RAP2.7 (RELATED TO AP2.7) DNA binding & Transcription factor & AT2G28550 (16.639) & 1.5 \\
\hline \multirow[t]{2}{*}{ miR319 } & TCP10 (TCP Domain Protein 10) & Transcription factor & AT2G31070 (10.122) & 3.5 \\
\hline & TCP4 (TCP family transcription factor 4) & Transcription factor & AT3G15030 (13.479) & 3.5 \\
\hline miR396 & AtGRF4 (Growth regulating factor 4) & Transcription activator & AT3G52910 (14.357) & 2 \\
\hline $\operatorname{miR} 408$ & TIL1 (TILTED 1); DNA binding/DNA-directed DNA polymerase/nucleic acid binding/nucleotide binding/zinc ion binding & Transcription factor & AT1G08260 (15.881) & 4 \\
\hline miR414 & WRKY DNA -binding domain & Transcription factor & AT4G31550 & \\
\hline
\end{tabular}

(UPE; Maximum energy to unpair the target site, UPE range: $0.0-25.0$; E-value range: $0.0-4.0$ ) 


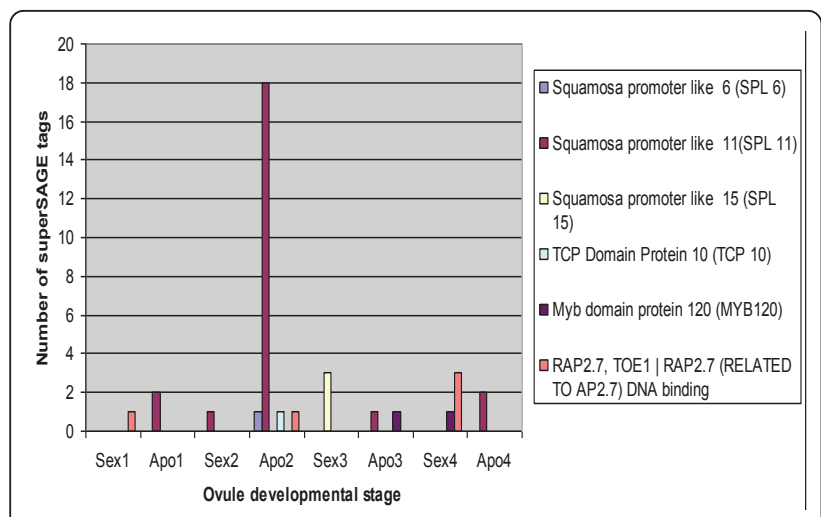

Figure 9 Expression of transcription factors across Boechera sexual and apomictic ovule developmental stages. Refer to Sharbel et al. [40] for descriptions of ovule stages.

TF expression could be associated with the penetrance of the apomictic phenotype, which has been shown to be genotype-specific in Boechera [63].

\section{Conclusions}

This study constitutes the first extensive insight into the conservation and expression of miRNAs in Boechera sexual and apomictic species. Of the expressed miRNA transcription factor targets observed, only the miR156/ 157 family target squamosa promoter binding proteinlike 11 (SPL11) was found differentially expressed with significant $(\mathrm{p} \leq 0.05)$ up-regulation at the stage two of ovule development in apomictic species. Also demonstrated here is that nucleotide changes in mature miRNAs significantly ( $\mathrm{p} \leq 0.05)$ enhance the thermodynamic stability of pre-miRNA stem-loops. This work will enhance subsequent elucidation of the repertoire of

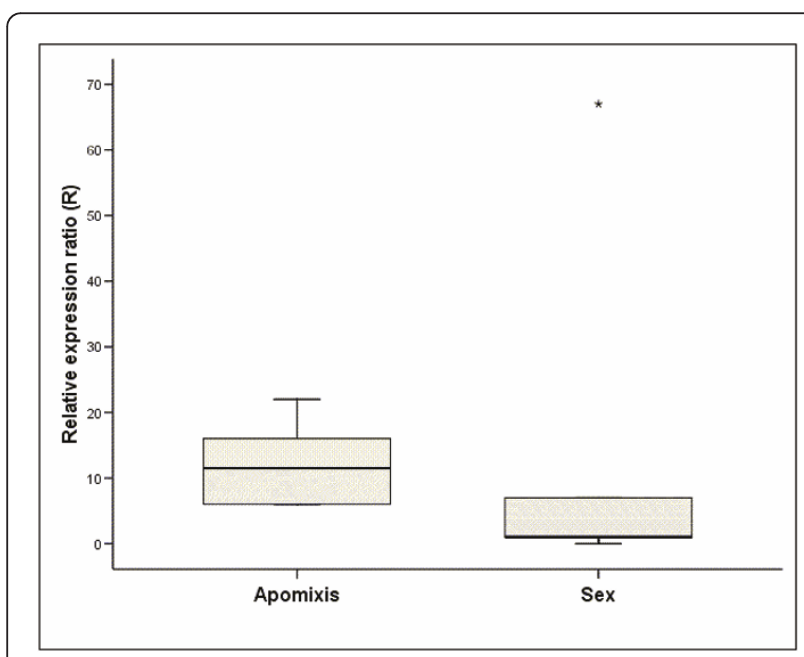

Figure 10 Relative differential expression of SPL11 transcription factor in stage two of apomictic and sexual ovules.
miRNA expression in Boechera towards revealing the potential role of miRNAs in the switch from sexual to apomictic reproduction.

\section{Methods}

Flower-specific Boechera 454 cDNA libraries used

Floral cDNA libraries used in this study are those previously reported by Sharbel et al. [39,40]. These libraries were sequenced from pooled flower stages 1-12 [64] of three diploid sexual plants (Accessions ES910-2-2 K, 105.6-1 $\mathrm{K}$ and B07261) and three apomictic plants (Accessions 67.5-K, 300.6.1-1 K and 218.2-2 K).

\section{Conserved miRNA reference set for bioinformatics procedures}

A total of 9274 previously reported non-redundant 2124 nucleotides long miRNAs (including their precursor sequences) collected from 121 plant species were obtained from the Plant MicroRNAs Database (PMRD as of February 8, 2011; [65]). These miRNAs were defined as a reference set of miRNA sequences for the identification of potentially conserved miRNAs in Boechera. To avoid redundant miRNAs, duplicated miRNAs shared between different species within the database were removed. In all, 8433 non-redundant miRNAs were obtained, and these were used as query sequences for a BLASTn search against all original 454 sequence reads from the apomictic and sexual Boechera libraries.

\section{Identification of conserved miRNAs}

The bioinformatics approaches used for identification of conserved miRNAs in Boechera species are outlined in Figure 1. The length of the EST sequences used to search for conserved miRNAs ranged between 51 and 478 nucleotides, with about $80 \%$ of them around 200 nucleotides long. In order to exclude all ESTs having exact matches to tRNA or rRNA sequences from further BLASTn searches, the sexual and apomictic EST libraries were first queried against ribosomal RNAs database from Rfam (http://www.sanger.ac.uk/Software/ Rfam/) and the Arabidopsis transfer RNAs database (http://lowelab.ucsc.edu/GtRNAdb/Athal/). Rather than using the miRNA precursors for BLASTn searches against our databases, the analysis was based mainly on the mature miRNA sequences considering that only mature miRNAs are highly conserved in plants $[42,43]$. The following BLASTn parameters which gave the highest and most reliable number of hits (blastall -p blastn -m 8 -e 1 -W 7 -r 1 -q -1 -i) were used. All resulting EST sequences with an alignment length of 20-24 nucleotides, three or fewer mismatches and no gaps compared to previously identified plant miRNAs were selected and compared with each other to eliminate redundancies. The obtained non-redundant sequences 
were then used for the prediction of secondary structures and screening for miRNA precursor sequences. The secondary structures of pre-miRNAs were generated using the Mfold 3.2 software, which is based on Zuker folding algorithm principles [48].

The secondary structure of candidate pre-miRNA sequences were analysed and scored for their potential to form miRNA precursors. A stem-loop was selected as a candidate miRNA precursor if it satisfied most of the following generally accepted criteria: (1) the mature miRNA is 20-24nt with a maximum of three mismatches compared with the corresponding known miRNA in other plant species; (2) the miRNA precursor (pre-miRNA) sequence folds into a stable hairpin structure such that one arm of the hairpin contains the mature miRNA sequence; (3) the predicted secondary structure of the pre-miRNA has lower minimal free energy (MFE $\leq-10 \mathrm{kcal} / \mathrm{mol}$ ) and minimal free energy index (MFEI) than other types of RNA (e.g. tRNA, rRNA); (4) the predicted mature miRNA has an $\mathrm{A}+\mathrm{U}$ content of 40-70\%; and (5) no loop or gap in the mature miRNA sequences [41].

\section{Microarray validation of conserved plant miRNAs}

The bioinformatically-identified miRNAs in floral tissues were further verified using a proprietary microarray analysis with LC Sciences, USA. The microarray assay was performed using 4 to $8 \mu \mathrm{g}$ total RNA sample from pooled flower tissues of sexual and apomictic genotypes. The total RNA was size fractionated using a YM-100 Microcon centrifugal filter (Millipore) and the isolated small RNAs (<300 nt) were 3'-extended with a poly(A) tail using poly(A) polymerase. An oligonucleotide tag was then ligated to the poly(A) tail for later fluorescent dye staining, and two different tags were used for two RNA samples in dual-sample experiments. Hybridization was performed overnight on a $\mu$ Paraflo microfluidic chip [spotted with all known plant mature miRNAs that were available in miRBase Release 14 (total 1117 unique mature miRNAs) and the Plant miRNA Database (total 5690 unique mature miRNAs)] using a micro-circulation pump (Atactic Technologies; [66]). On the microfluidic chip, each detection probe consisted of a chemically modified nucleotide coding segment complementary to a target miRNA, and a spacer segment of polyethylene glycol to extend the coding segment away from the substrate. The detection probes were made by in situ synthesis using PGR (photogenerated reagent) chemistry. The hybridization melting temperatures were balanced by chemical modifications of the detection probes, and hybridization was performed using $100 \mu \mathrm{L} 6 \times$ SSPE buffer $\left(0.90 \mathrm{M} \mathrm{NaCl}, 60 \mathrm{mM} \mathrm{Na} 2 \mathrm{HPO}_{4}, 6 \mathrm{mM}\right.$ EDTA, $\mathrm{pH}$ 6.8) containing $25 \%$ formamide at $34^{\circ} \mathrm{C}$. After RNA hybridization, tag-conjugating $\mathrm{Cy} 3$ and $\mathrm{Cy} 5$ dyes were circulated through the microfluidic chip for dye staining. The fluorescence data were collected on an Axon GenePix 4000B Microarray Scanner, and then analysed by first subtracting the background followed by normalization of the signals using a LOWESS filter (Locally-weighted Regression; [67]). A detectable miRNA on the array was identified if its signal intensity was higher than $3 \times$ (background standard deviation) and spot $C V<0.5$, and $p<0.01$ for the difference between $\mathrm{Cy} 3$ and Cy5 signals (LC Sciences).

\section{Prediction of Boechera gene targets of miRNA families}

A BLASTn search (blastall -p blastn -m 8 -e 1 -W 7 -r 1 -q $-1-\mathrm{i}$ ) was employed to detect complementarity between the validated miRNAs and predicted target ESTs in sexual and apomictic Boechera (Additional file 3, Table S1). Putative miRNA targets were identified based on the total numbers of mismatched nucleotides between miRNAs and the alignment structures of potential targets. To identify potential regulatory targets, a BLAST search (blastall -p blastn -m 8 -e 1 -W 7 -r 1 $-\mathrm{q}-1-\mathrm{i}$ ) was performed using the validated (from LC Sciences) conserved Boechera miRNAs against the $A$. thaliana protein-coding nucleotide databases (TAIR9 cDNA) using the miRU web server [68] from the Arabidopsis Information Resource (TAIR). The total number of allowed mismatches at complementary sites between miRNA sequences and potential mRNA targets in Arabidopsis were limited to a maximum of three, and no gaps were allowed at complementary sites. Finally, the Boechera homologues of potential targeted genes in Arabidopsis were chosen using a BLAST search (blastall -p blastn -m 8 -e 1 -W 7 -r 1 -q -1 -i) based on the degree of similarity of protein-coding mRNAs between A. thaliana and Boechera.

\section{Expression analysis of Transcription factor (TF) targets using SuperSAGE tags}

Finally, a comparative gene expression analysis of TF targets from 11 miRNA families was carried out. First, a BLASTn search using TF genes from Arabidopsis against the assembled Boechera EST database was performed (blastall -p blastn - $\mathrm{m} 8$-e $1-\mathrm{W} 7$-r 1 -q -1 -i). For each Arabidopsis TF, homologous Boechera TFs with an alignment having a bit score $\geq 100$ were selected. Next, 100\% sequence matches between the Boechera TFs and expression tags from 8 ovule-specific Boechera SuperSAGE libraries [40] were found using a BLASTn search. Finally, the expression patterns of the selected Boechera TFs corresponding to the obtained SuperSAGE tags were compared across four different ovule developmental stages between a sexual and apomictic Boechera genotype [40]. 


\section{Quantitative RT-PCR validation of differential SPL11 expression in ovule stage two of Boechera flowers}

Six accessions of apomictic and five of sexual Boechera were selected for the validation the differential expression of SPL11 (Additional file 4, Table S2). From these accessions, stage two ovules were micro-dissected, RNAs isolated and cDNAs prepared as described in Sharbel et al. [40]. The forward primer 5'-CAAAGT GCCCAAAAGTTACCGTGAGT-3' and reverse primer 5'-ACGCCTCGCATTATGATGAGAAAGA-3' with amplicon size of 137 nucleotides long were used for the qRT-PCR. Primers were designed avoiding intronic regions (to ensure the elimination of likely DNA contamination in samples) using the following parameters: temperature; $60^{\circ} \mathrm{C}, 20 \%<\mathrm{CG}$ content $<80 \%$, and PCR product size $<150 \mathrm{bp}$. For the real-time PCR reactions, the SYBR Green PCR Master Mix (Applied Biosystems) was used. qRT-PCR amplifications were performed in a 7900 HT Fast RT-PCR system (Applied Biosystems) with the following temperature profile for SYBRgreen assays: initial denaturation at $90^{\circ} \mathrm{C}$ for $10 \mathrm{~min}$, followed by 40 cycles of $95^{\circ} \mathrm{C}$ for $15 \mathrm{~s}$, and $60^{\circ} \mathrm{C}$ for $1 \mathrm{~min}$. The $\mathrm{Ct}$, defined as the PCR cycle at which a statistically significant increase of reporter fluorescence is first detected, was used as a measure for the starting copy numbers of the target gene. The mean expression level and standard deviation for each set of three technical replicates for each cDNA was calculated. Relative quantitation and normalization of the amplified targets were performed by the comparative $\Delta \Delta \mathrm{Ct}$ method in reference to the expression levels of the housekeeping gene ubiquitin [69].

\section{Additional material}

Additional file 1: Boechera stem-loop structures. List of predicted premiRNA structures of conserved miRNAs identified in Boechera species.

Additional file 2: Boechera miRNA families. Grouping of miRNA families identified by bioinformatics and microarray assay.

Additional file 3: Predicted miRNA targets. Gene targets of conserved miRNA families in Boechera species.

Additional file 4: Boechera genotypes. Boechera genotypes used for qRT-PCR validation of differential SPL11 expression.

\section{List of abbreviations}

AGO, argonaute; ESTs, expressed sequence tags; GSS, genome survey sequences; miRNA, microRNA; miRISC, miRNA-induced silencing complex; MFE, minimum fold energy; MFEl, minimal fold energy index; AMFE, adjusted minimal fold energy; PMRD, plant miRNA database; TAIR, arabidopsis information resource; SBP, squamosa promoter binding protein; GRT-PCR, quantitative reverse transcription PCR.

\section{Acknowledgements}

This work was funded by the Apomixis Research Group, using basic level funding provided by the IPK, in addition to funding from the International Max Planck Research School (IMPRS), Jena. The authors would like to thank
Prof. I. Schubert for comments on the manuscript. Samuel Amiteye also expresses his profound gratitude to the International Max Planck Research School (IMPRS), Jena, Germany for his current PhD funding.

\section{Author details}

${ }^{1}$ Leibniz Institute of Plant Genetics and Crop Plant Research (IPK), Corrensstrasse 3, D-06466 Gatersleben, Germany. ${ }^{2}$ Max Planck Institute for Chemical Ecology, Department of Entomology, Genomics Research Group, Hans-Knöll-Strasse 8, D-07745 Jena, Germany.

\section{Authors' contributions}

SA: Project development, bioinformatics, microarray analysis and draft of manuscript. JMC: Project development, analytical supervision and sample preparation. HV: Project development and sample preparation. TFS: Project development, bioinformatics and draft of manuscript. All authors have read and approved the final manuscript.

Received: 6 May 2011 Accepted: 11 October 2011

Published: 11 October 2011

\section{References}

1. Spillane C, Curtis MD, Grossniklaus U: Apomixis technology developmentvirgin births in farmers' fields? Nature Biotechnology 2004, 22:687-691.

2. Delmotte F, Letterme M, Bonhomme J, Rispe C, Simon JC: Multiple routes to asexuality in an aphid species. Pro R SoC Lond B 2001, 268:2291-2299.

3. Simon JC, Delmotte F, Rispe C, Crease T: Phylogenetic relationships between parthenogens and their sexual relatives: the possible routes to parthenogenesis in animals. Biol J Linn Soc Lond 2003, 79:151-163.

4. Koch M, Bishop J, Mitchell-Olds T: Molecular systematics and evolution of Arabidopsis and Arabis. Plant Biology 1999, 1:529-537.

5. Böcher TW: Cytological and embryological studies in the amphiapomictic Arabis holboellii complex. Det Kongelige Danske Videnskabernes Selskab 1951, 6:1-59.

6. Koch MA, Dobeš C, Mitchell-Olds T: Multiple hybrid formation in natural populations: concerted evolution of the internal transcribed spacer of nuclear ribosomal DNA (ITS) in North American Arabis divaricarpa (Brassicaceae). Mol. Biol. Evol 2003, 20:338-350.

7. Kiefer C, Dobeš C, Sharbel TF, Koch MA: Phylogeographic structure of the chloroplast DNA gene pool in North American Boechera - A genus and continental-wide perspective. Molecular Phylogenetics and Evolution 2009, 52:303-311.

8. Koltunow AM, Grossniklaus U: Apomixis: A developmental perspective. Annu Rev Plant Biol 2003, 54:547-574.

9. Carman JG: Asynchronous expression of duplicate genes in angiosperms may cause apomixis, bispory, tetraspory, and polyembryony. Biol J Linn Soc Lond 1997, 61:51-94.

10. Allen E, Xie Z, Gustafson AM, Sung GH, Spatafora JW, Carrington JC: Evolution of microRNA genes by inverted duplication of target gene sequences in Arabidopsis thaliana. Nat Genet 2004, 36:1282-1290.

11. Felippes FF, Schneeberger K, Dezulian T, Hudson DH, Weigel D: Evolution of Arabidopsis thaliana microRNAs from random sequences. RNA 2008, 14(12):2455-2459.

12. Sunkar R, Zhu JK: MicroRNAs and short-interfering RNAs in plants. J Integr Plant Biol 2007, 49:817-826.

13. Jagadeeswaran G, Saini A, Sunkar R: Biotic and abiotic stress downregulate miR398 expression in Arabidopsis. Planta 2009, 229:1009-1014.

14. Chapman EJ, Prokhnevsky Al, Gopinath K, Dolja W, Carrington JC: Viral RNA silencing suppressors inhibit the microRNA pathway at an intermediate step. Genes and Development 2004, 18(10):1179-1186.

15. Mallory AC, Reinhart BJ, Jones-Rhoades MW: MicroRNA control of PHABULOSA in leaf development: importance of pairing to the microRNA 5' region. The EMBO Journal 2004, 23(16):3356-3364.

16. Palatnik JF, Allen E, Wu X, Schommer C, Schwab R, Carrington JC, Weigel D: Control of leaf morphogenesis by microRNAs. Nature 2003, 425:257-263.

17. Subramanian S, Fu Y, Sunkar R, Barbazuk WB, Zhu JK, Oliver Y: Novel and nodulation-regulated microRNAs in soybean roots. BMC Genomics 2009, 9:160.

18. Millar AA, Gubler F: The Arabidopsis GAMYBlike genes, MYB33 and MYB65, are MicroRNA-regulated genes that redundantly facilitate anther development. Plant Cell 2005, 17(3):705-721. 
19. Olmedo-Monfil V, Dura'n-Figueroa N, Arteaga-Va'zquez M, DemesaAre'valo E, Autran D, Grimanelli D, Slotkin RK, Martienssen RA, VielleCalzada JP: Control of female gamete formation by a small RNA pathway in Arabidopsis. Nature 2010.

20. Rhoades MW, Bartel DP: Computational identification of plant microRNAs and their targets, including a stress-induced miRNA. Mol Cell 2004, 14:787-799.

21. Han MH, Goud S, Song L, Fedoroff N: The Arabidopsis double-stranded RNA-binding protein HYL1 plays a role in microRNA-mediated gene regulation. Proc Natl Acad Sci USA 2004, 101:1093-1098.

22. Vazquez F, Gasciolli V, Crete P, Vaucheret $H$ : The nuclear dsRNA binding protein HYL1 is required for microRNA accumulation and plant development, but not posttranscriptional transgene silencing. Curr Biol 2004, 14:346-351.

23. Yang L, Liu Z, Lu F, Dong A, Huang H: SERRATE is a novel nuclear regulator in primary microRNA processing in Arabidopsis. Plant J 2006, 47:841-850.

24. Vaucheret $H$, Vazquez F, Crete P, Bartel DP: The action of ARGONAUTE1 in the miRNA pathway and its regulation by the miRNA pathway are crucial for plant development. Genes \& Dev 2004, 18:1187-1197.

25. Baumberger N, Baulcombe DC: Arabidopsis ARGONAUTE1 is an RNA slicer that selectively recruits microRNAs and short interfering RNAs. Proc Natl Acad Sci USA 2005, 102:11928-11933.

26. Rhoades MW, Reinhart BJ, Lim LP, Burge CB, Bartel B, Bartel DP: Prediction of plant microRNA targets. Cell 2002, 110:513-520.

27. Chen X: A miRNA as a translational repressor of APETALA2 in Arabidopsis flower development. Science 2004, 303:2022-2025.

28. Brodersen $P$, Sakvarelidze-Achard $L$, Bruun-Rasmussen $M$, Dunoyer $P$, Yamamoto YY, Sieburth L, Voinnet O: Widespread translational inhibition by plant miRNAs and siRNAs. Science 2008, 320(5880):1185-90.

29. Zhang B, Pan $X$, Anderson TA: Identification of 188 conserved maize microRNAs and their targets. FEBS J 2006, 580(15):3753-3762a.

30. Zhou ZS, Huang SQ, Yang ZM: Bioinformatic identification and expression analysis of new microRNAs from Medicago truncatula. Biochemical and Biophysical Research Communications 2008, 374(3):538-542.

31. Rajagopalan R, Vaucheret H, Trejo J, Bartel DP: A diverse and evolutionarily fluid set of microRNAs in Arabidopsis thaliana. Genes \& Dev 2006, 20:3407-3425.

32. Fahlgren N, Howell MD, Kasschau KD, Chapman EJ, Sullivan CM, Cumbie JS, Givan SA, Law TF, Grant SR, Dangl JL, Carrington JC: High-throughput sequencing of Arabidopsis microRNAs: Evidence for frequent birth and death of MIRNA genes. PLOS ONE 2007, 2:e219.

33. Yao Y, Guo G, Ni Z, Sunkar R, Du J, Zhu JK, and Sun Q: Cloning and characterization of microRNAs from wheat (Triticum aestivum L.). Genome Biol 2007, 8:R96.

34. Moxon S, Jing R, Szittya G, Schwach F, Pilcher RLR, Moulton V, Dalmay T: Deep sequencing of tomato short RNAs identifies microRNAs targeting genes involved in fruit ripening. Genome Res 2008, 18:1602-1609.

35. Sunkar R, Zhu JK: Novel and stress-regulated microRNAs and other small RNAs from Arabidopsis. Plant Cell 2004, 16:2001-2019.

36. Sunkar R, Girke T, Jain PK, Zhu JK: Cloning and characterization of microRNAs from rice. Plant Cell 2005, 17:1397-1411.

37. Amiteye S, Corral JM, Sharbel TF: Overview of the potential of microRNAs and their target gene detection for cassava (Manihot esculenta) improvement. African Journal of Biotechnology 2011, 10(14):2562-2573.

38. Zhao $L, K i m$, Dinh $T$, Chen $X$ : miR172 regulates stem cell fate and defines the inner boundary of APETALA3 and PISTILLATA expression domain in Arabidopsis floral meristems. The Plant Journal 2007, 51:840-849.

39. Sharbel TF, Voigt ML, Corral JM, Thiel T, Varshney A, Kumlehn J, Vogel H, Rotter B: Molecular signatures of apomictic and sexual ovules in the Boechera holboellii complex. Plant J 2009, 58:870-882.

40. Sharbel TF, Voigt ML, Corral JM, Galla G, Kumlehn J, Klukas C, Schreiber F, Vogel H, Rotter B: Apomictic and sexual ovules of Boechera display heterochronic global gene expression patterns. Plant Cell 2010, 22:655-671.

41. Zhang B, Pan X, Stellwag EJ: Identification of soybean microRNAs and their targets. Planta 2008, 229:161-182.

42. Zhang BH, Pan XP, Cox SB, Cobb GP, Anderson TA: Evidence that miRNAs are different from other RNAs. Cell Mol Life Sci 2006, 63:246-254.
43. Zhang B, Pan X, Cannon CH, Cobb GP, Anderson TA: Conservation and divergence of plant microRNA genes. The Plant Journal 2006, 46:243-259.

44. Seffens $W$, Digby D: mRNAs have greater negative folding free energies than shuffled or codon choice randomized sequences. Nucleic Acids Res 1999, 27:1578-1584.

45. Bonnet E, Wuyts J, Rouze P: Evidence that microRNA precursors, unlike other non-coding RNAs, have lower folding free energies than random sequences. Bioinformation 2004, 20:2911-2917.

46. Jaeger JA, Turner DH, Zuker M: Predicting optimal and suboptimal secondary structure for RNA, in "Molecular Evolution: Computer Analysis of Protein and Nucleic Acid Sequences". In Methods in Enzymology Edited by: Doolittle RF 1990, 183:281-306.

47. MCCaskill JS: The equilibrium partition function and base pair binding probabilities for RNA secondary structure. Biopolymers 1990, 29(67):1105-1119.

48. Zuker M: Mfold web server for nucleic acid folding and hybridization prediction. Nucleic Acids Res 2003, 31(13):3406-3415.

49. Colaiacovo M, Subacchi A, Bagnaresi P, Lamontanara A, Cattivelli L, Faccioli P: A computational-based update on microRNAs and their targets in barley (Hordeum vulgare L.). BMC Genomics 2010, 11:595.

50. Thakur V, Wanchana S, Xu M, Bruskiewich R, Quick WP, Mosig A, Zhu X: Characterization of statistical features for plant microRNA prediction. BMC Genomics 2011, 12:108.

51. Bonnet E, Wuyts J, Rouze P, de Peer W: Detection of 91 potential conserved plant microRNAs in Arabidopsis thaliana and Oryza sativa identifies important target genes. Proc Natl Acad Sci USA 2004, 101:11511-11516.

52. Zhang BH, Pan XP, Wang QL, Cobb GP, Anderson TA: Identification and characterization of new plant microRNAs using EST analysis. Cell Research 2005, 15:336-360.

53. Zhou M, Gu L, Li P, Song X, Wei L, Chen Z, Cao X: Degradome sequencing reveals endogenous small RNA targets in rice (Oryza sativa L., ssp. Indica). Front Biol 2010, 5(1):67-90.

54. Jones-Rhoades MW, Bartel DP, Bartel B: MicroRNAs and their regulatory roles in plants. Annu Rev Plant Biol 2006, 57:19-53.

55. Chiou TJ: The role of microRNAs in sensing nutrient stress. Plant Cell Environ 2007, 30:323-332.

56. Gandikota M, Birkenbihl RP, Hohmann S, Cardon GH, Saedler H, Huijser P: The miRNA156/157 recognition element in the $3^{\prime}$ UTR of the Arabidopsis SBP box gene SPL3 prevents early Flowering by translational inhibition in seedlings. Plant J 2007, 49:683-693.

57. Aukerman MJ, Sakai H: Regulation of flowering time and floral organ identity by a microRNA and its APETALA2-like target genes. Plant Cell 2003, 15:2730-2741.

58. Baker CC, Sieber P, Wellmer F, Meyerowitz EM: The early extra petals1 mutant uncovers a role for microRNA miR164c in regulating petal number in Arabidopsis. Curr Biol 2005, 15:303-315.

59. Kasschau KD, Xie Z, Allen E, Llave C, Chapman EJ, Krizan KA, Carrington JC: P1/HC-Pro, a viral suppressor of RNA silencing, interferes with Arabidopsis development and miRNA unction. Dev Cell 2003, 4:205-217.

60. Vierstra RD: The ubiquitin/26S proteasome pathway, the complex last chapter in the life of many plant proteins. Trends Plant Sci 2003, 8:135-142.

61. Kim JH, Choi D, Kende H: The AtGRF family of putative transcription factors is involved in leaf and cotyledon growth in Arabidopsis. Plant $J$ 2003, 36:94-104.

62. Grossniklaus U: From sexuality to apomixis: molecular and genetic approaches. In The Flowering of Apomixis: From Mechanisms to Genetic Engineering. Edited by: Savidan Y, Carman JG, Dresselhaus T. Mexico: CIMMYT, IRD European Commission DG VI (FAIR); 2001:168-211.

63. Aliyu OM, Schranz ME, Sharbel TF: Quantitative variation for apomixis components in the genus Boechera. Am J Bot 2010, 97(10):1719-1731.

64. Smyth DR, Bowman JL, Meyerowitz EM: Early flower development in Arabidopsis. Plant Cell 1990, 2:755-767.

65. Zhang Z, Yu J, Li D, Zhang Z, Liu F, Zhou X, Wang T, Ling Y, Su Z: PMRD: plant microRNA database. Nucleic Acids Research 2010, 38:D806-D813.

66. Gao X, Gulari E, Zhou X: In situ synthesis of oligonucleotide microarrays. Biopolymers 2004, 73:579-596.

67. Bolstad BM, Irizarry RA, Astrand M, Speed TP: A comparison of normalization methods for high density oligonucleotide array data based on variance and bias. Bioinformatics 2003, 19:185-193. 
68. Zhang Y: miRU: an automated plant miRNA target prediction server. Nucleic Acids Res 2005, , 33 Web Server: W701-W704.

69. Pellino M, Sharbel TF, Mau M, Amiteye S, Corral JM: Selection of reference genes for quantitative real-time PCR expression studies of microdissected reproductive tissues in apomictic and sexual Boechera. BMC Research Notes 2011, 4:303.

\section{doi:10.1186/1471-2164-12-500}

Cite this article as: Amiteye et al: Analysis of conserved microRNAs in floral tissues of sexual and apomictic Boechera species. BMC Genomics 2011 12:500.

Submit your next manuscript to BioMed Central and take full advantage of:

- Convenient online submission

- Thorough peer review

- No space constraints or color figure charges

- Immediate publication on acceptance

- Inclusion in PubMed, CAS, Scopus and Google Scholar

- Research which is freely available for redistribution

Submit your manuscript at www.biomedcentral.com/submit 